\title{
Quantum Semiparametric Estimation
}

\author{
Mankei Tsang $\odot,{ }^{1,2, *}$ Francesco Albarelli, ${ }^{3,4, \dagger}$ and Animesh Datta $\odot^{4, \$}$ \\ ${ }^{1}$ Department of Electrical and Computer Engineering, National University of Singapore, \\ 4 Engineering Drive 3, Singapore 117583 \\ ${ }^{2}$ Department of Physics, National University of Singapore, 2 Science Drive 3, Singapore 117551 \\ ${ }^{3}$ Faculty of Physics, University of Warsaw, 02-093 Warszawa, Poland \\ ${ }^{4}$ Department of Physics, University of Warwick, Coventry CV4 7AL, United Kingdom
}

(Received 1 August 2019; revised 8 May 2020; accepted 1 June 2020; published 30 July 2020)

\begin{abstract}
In the study of quantum limits to parameter estimation, the high dimensionality of the density operator and that of the unknown parameters have long been two of the most difficult challenges. Here, we propose a theory of quantum semiparametric estimation that can circumvent both challenges and produce simple analytic bounds for a class of problems in which the dimensions are arbitrarily high, few prior assumptions about the density operator are made, but only a finite number of the unknown parameters are of interest. We also relate our bounds to Holevo's version of the quantum Cramér-Rao bound, so that they can inherit the asymptotic attainability of the latter in many cases of interest. The theory is especially relevant to the estimation of a parameter that can be expressed as a function of the density operator, such as the expectation value of an observable, the fidelity to a pure state, the purity, or the von Neumann entropy. Potential applications include quantum state characterization for many-body systems, optical imaging, and interferometry, where full tomography of the quantum state is often infeasible and only a few select properties of the system are of interest.
\end{abstract}

DOI: 10.1103/PhysRevX.10.031023

Subject Areas: Quantum Information

\section{INTRODUCTION}

The random nature of quantum mechanics has practical implications for the noise in sensing, imaging, and quantum-information applications [1-6]. To derive their fundamental quantum limits, one standard approach is to compute quantum versions of the Cramér-Rao bound [1-5,7-9]. In addition to serving as rigorous limits to parameter estimation, the quantum bounds have inspired new sensing and imaging paradigms that go beyond conventional methods [3-5].

The study of quantum limits has grown into an active research field called quantum metrology in recent years, building on the pioneering work of Helstrom [1] and Holevo [7]. A major current challenge is the computation of quantum bounds for high-dimensional density operators and high-dimensional parameters, as the brute-force method quickly becomes intractable for increasing dimensions; see Refs. $[10,11]$ for a sample of recent efforts to

\footnotetext{
*mankei@nus.edu.sg; https://blog.nus.edu.sg/mankei/

†rancesco.albarelli@gmail.com

*animesh.datta@warwick.ac.uk
}

Published by the American Physical Society under the terms of the Creative Commons Attribution 4.0 International license. Further distribution of this work must maintain attribution to the author(s) and the published article's title, journal citation, and DOI. combat the so-called curse of dimensionality. Most of the existing methods, however, ultimately have to resort to numerics for high dimensions. While numerical methods are no doubt valuable, analytic solutions should be prized more highly — as with any study in physics—for their simplicity and offer of insights. Unfortunately, except for a few cases where one can exploit the special structures of the density-operator family [1,7,12-15], analytic results for high-dimensional problems remain rare in quantum metrology.

Here, we propose a theory of quantum semiparametric estimation that can turn the problem on its head and deal with density operators with arbitrarily high dimensions and little assumed structure. The theory is especially relevant to the estimation of a parameter that can be expressed as a function of the density operator, such as the expectation value of an observable, the fidelity to a given pure state, the purity, or the von Neumann entropy. The density operator is assumed to come from an enormous family, its dimension can be arbitrarily high and possibly infinite, and the unknown "nuisance" parameters have a similar dimension to that of the density operator. Despite the seemingly bleak situation, our theory can yield surprisingly simple analytic results, precisely because of the absence of structure. Our results are ideally suited to scientific applications, such as quantum state characterization [16-18], optical imaging $[1,5,6,14,15]$, and interferometry $[1-3,7]$, where 
the dimensions can be high, the density operator is difficult to specify fully, and it is prudent to assume little prior information.

The theory set forth generalizes the deep and exquisite theory of semiparametric estimation in classical statistics [19-21], which has seen wide applications in fields such as biostatistics [21], econometrics [22], astrostatistics [23], and, most recently, optical superresolution [24]. By necessity, the classical theory involves infinite-dimensional spaces for random variables and makes extensive use of geometric and Hilbert-space concepts. As seen later, the operator Hilbert space introduced by Holevo [7,25] turns out to be the right arena for the quantum case, and the geometric picture of quantum states $[8,9,26,27]$ can provide illuminating insights.

Our formalism is primarily based on Helstrom's version of the quantum Cramér-Rao bound [1]. While this approach allows us to adapt the classical methods more easily, it is unable to account for the increased errors due to the incompatibility of quantum observables when multiple parameters are involved [7,28]. We address this issue by studying also Holevo's version of the quantum Cramér-Rao bound [7] in the semiparametric setting and proving that the two versions turn out to be close. This result enables our bounds to inherit the asymptotic attainability of Holevo's bound [28-30] in many cases of interest.

\section{PREVIEW OF TYPICAL RESULTS}

Before going into the formalism, we present some typical results of the theory to offer motivation.

Suppose that an experimenter receives $N$ quantum objects, such as atoms, electrons, photons, or optical pulses, each with the same quantum state $\rho$. The experimenter would like to estimate a parameter $\beta$ as a function of $\rho$. Without any knowledge or assumption about $\rho$, what is the best measurement to perform for the estimation of $\beta$, and what is the fundamental limit to the precision for any measurement?

The quantum semiparametric theory can provide simple answers to the above questions. For the simplest example, let $\beta=\operatorname{tr} \rho Y$, where $Y$ is a given observable, and assume that the estimator is required to be unbiased. For example, one may wish to estimate

(1) the mean position of photons or electrons in optical or electron microscopy,

(2) the mean photon number in an optical mode in optical sensing, imaging, and communication [1],

(3) the mean energy, momentum, or field of quantum particles in particle-physics, condensed-matter, or quantum-chemistry experiments, or

(4) a density-matrix element, the fidelity $\langle\psi|\rho| \psi\rangle$ to a target pure state $|\psi\rangle\rangle$, or an entanglement witness in quantum information experiments [16,17].

This problem appears in all areas of quantum mechanics [31], as most quantum calculations offer predictions in terms of expectation values only, and experiments that aim to estimate the expectation values and verify the predictions with few assumptions about the density operator are, in essence, semiparametric estimation. The theory here shows that the optimal measurement is simply a von Neumann measurement of the observable $Y$ of each copy of the objects, followed by an average of the outcomes. For any measurement, the mean-square error of the estimation, denoted by the sans serif $\mathrm{E}$, has a quantum limit given by

$$
\mathrm{E} \geq \frac{1}{N} \operatorname{tr} \rho(Y-\beta)^{2}
$$

Absent any information about $\rho$, the separate measurements and the sample mean seem to be the most obvious procedure, but it is not at all obvious that it is optimal, given the infinite possibilities allowed by quantum mechanics.

While Eq. (2.1) has been derived before via a more conventional method for a finite-dimensional $\rho$ [32], our theory can also deal with infinite dimensions as well as more advanced examples in quantum information and quantum thermodynamics. For example, if the parameter of interest is the purity $\beta=\operatorname{tr} \rho^{2}$, the bound is

$$
\mathrm{E} \geq \frac{4}{N} \operatorname{tr} \rho(\rho-\beta)^{2}
$$

and if the parameter is the relative entropy $\beta=\operatorname{tr} \rho(\ln \rho-$ $\ln \sigma$ ) with respect to a target state $\sigma$, the bound is

$$
\mathrm{E} \geq \frac{1}{N} \operatorname{tr} \rho(\ln \rho-\ln \sigma-\beta)^{2} .
$$

For these two examples, the bounds are asymptotically attainable in principle, at least when $\rho$ is finite dimensional [28-30].

The semiparametric theory is relevant to experiments on many-body quantum systems and quantum simulation [33], because often there is no simple model for $\rho$, full tomography of $\rho$ is infeasible, and only a few select properties of the system may be of interest. Although significant literature in quantum information has been devoted to such semiparametric problems [16-18], their connections to the classical theory have not yet been recognized. By generalizing the classical theory, this work establishes fundamental limits to the task, indicating the minimum amount of resources needed to achieve a desired precision and also offering a rigorous yardstick for experimental design. This work thus addresses a foundational question by Horodecki [18]: "What kind of information (whatever it means) can be extracted from an unknown quantum state at a small measurement cost?" Our work shows that quantum metrology — and quantum semiparametric estimation, in particular - offers a viable attack on the question via a statistical notion of efficiency. 
An extension of the above scenario is the estimation of $\beta$ given a constraint on $\rho$. For example, suppose that the quantum state is known to possess a mean energy $\operatorname{tr} \rho H=E$, where $H$ is the Hamiltonian, or attain a fidelity of $\langle\phi|\rho| \phi\rangle=F$ with respect to another pure state $|\phi\rangle$. How may this new information affect the estimation? Write the constraint as $\operatorname{tr} \rho Z=\zeta$, where $Z$ is an observable and $\zeta$ is a given constant. The quantum bound for the $\beta=\operatorname{tr} \rho Y$ example turns out to be

$$
\begin{array}{cc}
\mathrm{E} \geq \frac{1}{N}\left(V_{Y}-\frac{C_{Y Z}^{2}}{V_{Z}}\right), & V_{Y}=\operatorname{tr} \rho(Y-\beta)^{2}, \\
C_{Y Z}=\operatorname{tr} \rho(Y-\beta) \circ(Z-\zeta), & V_{Z}=\operatorname{tr} \rho(Z-\zeta)^{2},
\end{array}
$$

where $A \circ B=(A B+B A) / 2$ denotes the Jordan product. The bound is reduced by the correlation between $Y$ and $Z$.

Another paradigmatic problem in quantum metrology is displacement estimation [1-3,7], which can be modeled by

$$
\rho=\exp (-i H \beta) \rho_{0} \exp (i H \beta),
$$

where $\rho_{0}$ is the initial state, $H$ is a generator, such as the photon-number operator in optical interferometry, and $\beta$ is the displacement parameter to be estimated. Applications range from optical and atomic interferometry to atomic clocks, magnetometry, laser ranging, and localization microscopy $[2,3,6]$. If nothing is known about $\rho_{0}$ other than a constraint $\operatorname{tr} \rho_{0} Z=0$, the quantum bound turns out to be

$$
\mathrm{E} \geq \frac{\operatorname{tr} \rho_{0} Z^{2}}{N\left\{-i \operatorname{tr} \rho_{0}[Z, H]\right\}^{2}},
$$

where $[Z, H] \equiv Z H-H Z$. Our theory can, in fact, give similarly simple results for a class of such semiparametric problems.

It must be stressed that, apart from the underlying Hilbert space and the constraints discussed above, the experimenter is assumed to know nothing about the density operator, and the bounds here are valid regardless of its dimension. The existing method of deriving such quantum limits is to model $\rho$ with many parameters $[8,9,29,32]$, compute a quantum version of the Fisher information matrix, and then invert it. This brute-force method is rarely feasible for problems with high or infinite dimensions. A new philosophy is needed.

In the next sections, we present the theory of quantum semiparametric estimation in increasing sophistication. Sections III and IV generalize the quantum Cramér-Rao bound proposed by Helstrom [1] in a geometric picture. While the picture is not new $[9,26]$, it has so far remained an intellectual curiosity only. Sections III and IV show that it can, in fact, give simple solutions, such as Eqs. (2.1)-(2.3), to a class of semiparametric problems with arbitrary dimensions. Section III establishes the general formalism and also proves results that are valid for finite dimensions, while Sec. IV deals with the infinite-dimensional case via an elegant concept called parametric submodels. In the classical theory, the concept was first adumbrated by Stein [34] and developed by Levit and many others [19-21]. Section V further develops the formalism to account for constraints on the density-operator family, in order to produce results such as Eq. (2.4). An example of entropy estimation in quantum thermodynamics is also discussed there. Section VI discusses some practical problems in optics and summarizes existing results on incoherent optical imaging [5] in the language of quantum semiparametrics, in order to provide a more concrete context for the formalism. Section VII considers semiparametric estimation in the presence of explicit nuisance parameters and studies, in particular, the problem of displacement estimation with a poorly characterized initial state, in order to produce results such as Eq. (2.7). To complete the formalism, Sec. VIII considers a vectoral parameter of interest and Holevo's version of the quantum Cramér-Rao bound [7]. There, we prove that the Helstrom and Holevo bounds are equal if the parameter of interest is a scalar, and they remain within a factor of 2 of each other in the vectoral case. The latter fact generalizes a recent result in the parametric setting [35]. Thus, the Helstrom version can inherit the asymptotic attainability of the latter [28-30] to within a factor of 2 .

\section{GEOMETRIC PICTURE OF QUANTUM ESTIMATION THEORY}

This section is organized as follows. Section III A introduces the Helstrom bound in the conventional formulation. Section III B introduces some important Hilbert-space concepts, including the tangent space and the influence operators. Section IIIC generalizes the Helstrom bound in terms of a projection of an influence operator into the tangent space. Section III D shows how an influence operator can be derived for a given parameter of interest, while Sec. III E proves that the tangent space is simple if the density operator is assumed to be finite dimensional but otherwise arbitrary. The projection is then straightforward, and Sec. III E demonstrates the derivation of Eqs. (2.1)-(2.3) as examples.

\section{A. Helstrom bound}

Let

$$
\mathbf{F} \equiv\left\{\rho(\theta): \theta \in \Theta \subseteq \mathbb{R}^{p}\right\}
$$

be a family of density operators parametrized by $\theta=$ $\left(\theta_{1}, \ldots, \theta_{p}\right)^{\top}$, where the superscript $\top$ denotes the matrix transpose and $p$ denotes the dimension of the parameter space $\Theta$. The operators are assumed to operate on a common Hilbert space $\mathcal{H}$, with an orthonormal basis 


$$
\left\{|j\rangle: j \in \mathcal{Q},\langle j \mid k\rangle=\delta_{j k}\right\}
$$

that does not depend on $\theta$. Let

$$
d \equiv \operatorname{dim} \mathcal{H}=|\mathcal{Q}|
$$

be the dimension of $\mathcal{H}$, which may be infinite. The family is assumed to be smooth enough so that any $\partial_{j} \equiv \partial / \partial \theta_{j}$ can be interchanged with the operator trace tr in any operation on $\rho(\theta)$. Define $\partial \equiv\left(\partial_{1}, \ldots, \partial_{p}\right)^{\top}$, and define a vector of operators $S \equiv\left(S_{1}, \ldots, S_{p}\right)^{\top}$ as solutions to

$$
\partial \rho=\rho \circ S,
$$

which is shorthand for the system of equations

$$
\left.\partial_{j} \rho(\theta)\right|_{\theta=\phi}=\rho(\phi) \circ S_{j}(\phi), \quad j=1, \ldots, p .
$$

$\phi$ is the true parameter value, and all functions of $\theta$ in this section are assumed to be evaluated implicitly at the same $\theta=\phi$. Each $S_{j}$ is called a symmetric logarithmic derivative in the quantum metrology literature, but here we call it a score, in accordance with the statistics terminology [19-21]. All vectors are assumed to be column vectors in this paper.

To model a measurement, define a positive operatorvalued measure (POVM) $E$ on a measurable space $\left(\mathcal{X}, \Sigma_{\mathcal{X}}\right)$, where $\Sigma_{\mathcal{X}}$ is the sigma algebra on the set $\mathcal{X}$. Let the parameter of interest be a scalar $\beta(\theta) \in \mathbb{R}$; generalization for a vectoral $\beta$ is done in Sec. VIII. Assume an estimator $\check{\beta}: \mathcal{X} \rightarrow \mathbb{R}$ that satisfies

$$
\int \check{\beta}(\lambda) \operatorname{tr} d E(\lambda) \rho=\beta, \quad \int \check{\beta}(\lambda) \operatorname{tr} d E(\lambda) \partial \rho=\partial \beta .
$$

$(E, \check{\beta})$ is called a locally unbiased measurement, as we require Eqs. (3.6) to hold only at the true $\theta=\phi$. Only local unbiasedness conditions are needed in this paper, and for brevity we no longer explicitly describe them as local. Define the mean-square estimation error as

$$
\mathrm{E} \equiv \int[\check{\beta}(\lambda)-\beta]^{2} \operatorname{tr} d E(\lambda) \rho
$$

If $p<\infty$, a quantum version of the Cramér-Rao bound due to Helstrom [1], denoted by the sans serif $\mathrm{H}$, applies to any unbiased measurement and can be expressed as

$$
\mathrm{E} \geq \mathrm{H} \equiv(\partial \beta)^{\top} K^{-1} \partial \beta,
$$

where the Helstrom information matrix $K$ is defined as

$$
K_{j k} \equiv \operatorname{tr} \rho\left(S_{j} \circ S_{k}\right)
$$

The Helstrom bound sets a lower bound on the estimation error for any quantum measurement and any unbiased estimator [1,7-9]. The estimation of $\beta$ with an infinitedimensional $\theta(p=\infty)$ is called semiparametric estimation in statistics [19-21], although the methodology applies to arbitrary dimensions. If $\theta$ is partitioned into $\left(\beta, \eta_{1}, \eta_{2}, \ldots\right)^{\top}$, then $\eta$ is called nuisance parameters $[21,36]$.

\section{B. Hilbert spaces for operators}

We now follow Holevo [7,25] and introduce operator Hilbert spaces in order to generalize the Helstrom bound for semiparametric estimation. The formalism may seem daunting at first sight, but the payoff is substantial, as it simplifies proofs, treats the infinite-dimensional case rigorously, and also enables one to avoid the explicit computation of $S$ and $K^{-1}$ for a large class of problems. In the following, we assume familiarity with the basic theory of Hilbert spaces and the mathematical treatment of quantum mechanics; see, for example, Refs. [7,37,38].

All operators considered in this paper are self-adjoint. Consider $\rho$ in the diagonal form $\rho=\sum_{j} \lambda_{j}\left|e_{j}\right\rangle\left\langle e_{j}\right|$ with $\lambda_{j}>0$. The support of $\rho$ is $\operatorname{supp}(\rho)=\operatorname{span}\left\{\left|e_{j}\right\rangle\right\} \subseteq \mathcal{H}$, where span denotes the closed linear span. $\rho$ is called full rank if $\operatorname{supp}(\rho)=\mathcal{H}$. Define the weighted inner product between two operators $h$ and $g$ as

$$
\langle h, g\rangle \equiv \operatorname{tr} \rho(h \circ g)
$$

and a norm as

$$
\|h\| \equiv \sqrt{\langle h, h\rangle}
$$

not to be confused with the operator norm $\|h\|_{\text {op }}=$ $\sup _{|\psi\rangle \in \mathcal{H}} \sqrt{\left\langle\psi\left|h^{2}\right| \psi\right\rangle} \geq\|h\|$. An operator is called bounded if $\|h\|_{\text {op }}<\infty$ and square summable with respect to $\rho$ if $\|h\|<\infty$, although all operators are bounded by definition if $d<\infty$. For two vectors of operators $A$ and $B$, it is convenient to use $\langle A, B\rangle$ to denote a matrix with entries

$$
\langle A, B\rangle_{j k}=\left\langle A_{j}, B_{k}\right\rangle,
$$

such as $K=\langle S, S\rangle$ as a Gram matrix.

Define the real Hilbert space for square-summable operators with respect to the true $\rho$ as $[7,25]$

$$
\mathcal{Y} \equiv\{h:\|h\|<\infty\} .
$$

To be precise, each Hilbert-space element is an equivalence class of operators with zero distance between them, viz., $\left\{\hat{h}_{j}:\left\|\hat{h}_{j}-\hat{h}_{k}\right\|=0 \forall j, k\right\}$. The distinction between an element and its operators is important only if $\rho$ is not full rank; we put a hat on an operator if the distinction is called for. Two important Hilbert-space elements are the identity 
element $I$ and the zero element 0 ; sometimes, we substitute $I=1$ for brevity.

Define a subspace of zero-mean operators as

$$
\mathcal{Z} \equiv\{h \in \mathcal{Y}: \operatorname{tr} \rho h=\langle h, I\rangle=0\}
$$

and the orthocomplement of $\mathcal{Z}$ in $\mathcal{Y}$ as

$$
\mathcal{Z}^{\perp} \equiv\{h \in \mathcal{Y}:\langle g, h\rangle=0 \forall g \in \mathcal{Z}\}=\operatorname{span}\{I\} .
$$

In particular, the projection of any $h \in \mathcal{Y}$ into $\mathcal{Z}^{\perp}$ is simply $\Pi\left(h \mid \mathcal{Z}^{\perp}\right)=\langle h, I\rangle$, where $\Pi$ denotes the projection map, and

$$
\Pi(h \mid \mathcal{Z})=h-\Pi\left(h \mid \mathcal{Z}^{\perp}\right)=h-\langle h, I\rangle .
$$

The most important Hilbert space in estimation theory is the tangent space spanned by the set of scores $\{S\} \equiv$ $\left\{S_{1}, \ldots, S_{p}\right\}$ [19-21], generalized here as

$$
\mathcal{T} \equiv \overline{\operatorname{span}}\{S\} \subseteq \mathcal{Z}
$$

$\{S\}$ is also known as the tangent set. The condition $\mathcal{T} \subseteq \mathcal{Z}$ requires the assumption $K_{j j}=\left\langle S_{j}, S_{j}\right\rangle<\infty$ for all $j$; the zero-mean requirement is satisfied because $\langle S, I\rangle=$ $\operatorname{tr} \partial \rho=\partial \operatorname{tr} \rho=0$. A useful relation for any bounded operator $h$ is

$$
\left\langle S_{j}, h\right\rangle=\operatorname{tr} \rho\left(S_{j} \circ h\right)=\operatorname{tr}\left(\rho \circ S_{j}\right) h=\operatorname{tr}\left(\partial_{j} \rho\right) h,
$$

via Eq. (2.8.88) in Ref. [7]. Denote also the orthocomplement of $\mathcal{T}$ in $\mathcal{Z}$ as

$$
\mathcal{T}^{\perp} \equiv\{h \in \mathcal{Z}:\langle S, h\rangle=0\}
$$

which is useful if a projection of $h \in \mathcal{Z}$ into $\mathcal{T}$ is desired and $\Pi\left(h \mid \mathcal{T}^{\perp}\right)$ is easier to compute, since

$$
\Pi(h \mid \mathcal{T})=h-\Pi\left(h \mid \mathcal{T}^{\perp}\right) .
$$

Another important concept in the classical theory is the influence functions [19-21], which we generalize by defining the set of influence operators as

$$
\mathcal{D} \equiv\{\delta \in \mathcal{Z}:\langle S, \delta\rangle=\partial \beta\}
$$

These operators play a major role in Holevo's formulation of quantum Cramér-Rao bounds [7,39], although their connection to the classical concept did not seem to be appreciated before.

\section{Generalized Helstrom bound}

Let the error operator with respect to an unbiased measurement be

$$
\delta=\int \check{\beta}(\lambda) d E(\lambda)-\beta .
$$

It can be shown (see Sec. 6.2 in Ref. [7]) that $\delta \in \mathcal{D}$ (as long as $\|\delta\|<\infty$ ) and also that $\|\delta\|^{2}$ bounds the estimation error as

$$
\mathrm{E} \geq\|\delta\|^{2} .
$$

A generalized Helstrom bound (GHB) for any unbiased measurement, denoted by $\tilde{\mathrm{H}}$, can then be expressed as

$$
\mathrm{E} \geq\|\delta\|^{2} \geq \inf _{\delta \in \mathcal{D}}\|\delta\|^{2} \equiv \tilde{\mathrm{H}} .
$$

We call an unbiased measurement efficient if it has an error that achieves the GHB, following the common statistics terminology [19-21].

Proofs that Eq. (3.24) is equal to Eq. (3.8) if $p<\infty$ and $K^{-1}$ exists can be found in Refs. [26,39,40]. The following theorem gives a more general expression that is the cornerstone of quantum semiparametric estimation.

Theorem 1.-

$$
\tilde{\mathrm{H}}=\min _{\delta \in \mathcal{D}}\|\delta\|^{2}=\left\|\delta_{\mathrm{eff}}\right\|^{2},
$$

where $\delta_{\text {eff }}$, henceforth called the efficient influence, is the unique element in the influence-operator set $\mathcal{D}$ given by

$$
\delta_{\text {eff }}=\Pi(\delta \mid \mathcal{T})
$$

and $\Pi(\delta \mid \mathcal{T})$ denotes the projection of any influence operator $\delta \in \mathcal{D}$ into the tangent space $\mathcal{T}$.

Proof.-The proof is similar to the classical one [20,21]. First note that, since $\mathcal{D} \subseteq \mathcal{Z}=\mathcal{T} \oplus \mathcal{T}^{\perp}$, any $\delta \in \mathcal{D}$ can always be decomposed into

$\delta=\delta_{\text {eff }}+h, \quad \delta_{\text {eff }}=\Pi(\delta \mid \mathcal{T}), \quad h=\Pi\left(\delta \mid \mathcal{T}^{\perp}\right)$.

This fact implies $\left\langle S, \delta_{\text {eff }}\right\rangle=\langle S, \delta-h\rangle=\langle S, \delta\rangle=\partial \beta$, and, therefore, $\delta_{\text {eff }} \in \mathcal{D}$. Now the Pythagorean theorem gives

$$
\|\delta\|^{2}=\left\|\delta_{\text {eff }}\right\|^{2}+\|h\|^{2} \geq\left\|\delta_{\text {eff }}\right\|^{2},
$$

which results in Eq. (3.25).

To prove the uniqueness of $\delta_{\text {eff }}$ in $\mathcal{D}$, suppose that there exists another $\delta^{\prime} \in \mathcal{D}$ that gives $\left\|\delta^{\prime}\right\|=\left\|\delta_{\text {eff }}\right\|$. Define $g=\delta^{\prime}-\delta_{\text {eff }}$. Since $\langle S, g\rangle=\left\langle S, \delta^{\prime}\right\rangle-\left\langle S, \delta_{\text {eff }}\right\rangle=\partial \beta-\partial \beta=0$, $g \in \mathcal{T}^{\perp}$, and the Pythagorean theorem yields $\left\|\delta^{\prime}\right\|^{2}=$ $\left\|\delta_{\text {eff }}\right\|^{2}+\|g\|^{2}$. This fact implies that $\|g\|=0$ and 


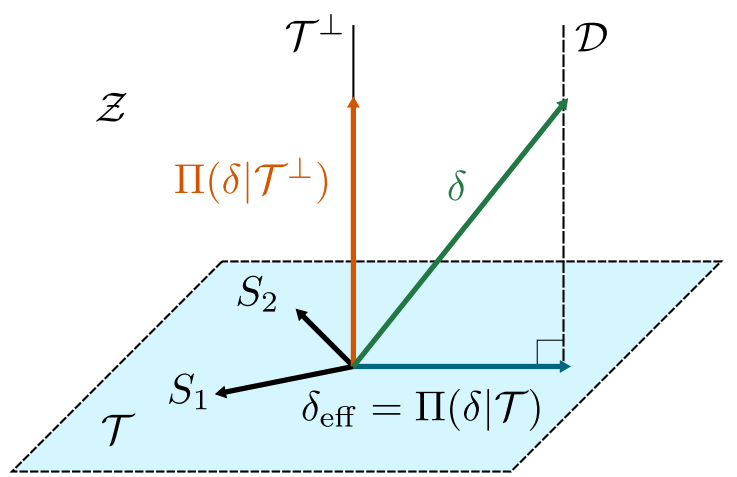

FIG. 1. The whole space in the picture represents $\mathcal{Z}$, the space of zero-mean operators. $\mathcal{T}$ is the tangent space spanned by the tangent set $\{S\} . \mathcal{T}^{\perp}$ is the orthocomplement, which contains elements orthogonal to all the scores. $\mathcal{D}$ is the set of influence operators, which all have a fixed projection in $\mathcal{T}$ determined by $\partial \beta . \delta$ is an influence operator in $\mathcal{D}$. The projection of $\delta$ into $\mathcal{T}$ gives the efficient influence $\delta_{\text {eff }}$, which has the smallest norm among all the influence operators. $\Pi\left(\delta \mid \mathcal{T}^{\perp}\right)$ is the projection of $\delta$ into $\mathcal{T}^{\perp}$.

$g=0$, contradicting the assumption that $\delta^{\prime} \neq \delta_{\text {eff }}$. Hence, $\delta_{\text {eff }}$ must be unique, and $\Pi(\delta \mid \mathcal{T})$ for any $\delta \in \mathcal{D}$ results in the same $\delta_{\text {eff }}$.

Figure 1 illustrates all the Hilbert-space concepts involved in Theorem 1 .

Before we apply the theorem to examples, we list a couple of important corollaries. The first corollary reproduces the original Helstrom bound given by Eq. (3.8) and is expected from earlier derivations; see, for example, Eq. (20) in Chap. 18 in Ref. [9] and Eq. (7.93) in Ref. [26]. Here, we simply clarify that it is a special case of Theorem 1.

Corollary 1.-If $p<\infty$ and $K^{-1}=\langle S, S\rangle^{-1}$ exists, the GHB is equal to the original Helstrom bound given by Eq. (3.8).

Proof.-The proof is delegated to the Appendix A.

Note that, unlike Eq. (3.8), which assumes that $S$ consists of linearly independent operators and $K$ is invertible, Theorem 1 works with no regard for any linear dependence in $S$. This generalization is, in fact, indispensable to the semiparametric theory, especially when the concept of parametric submodels is introduced in Sec. IV.

The second corollary, which gives a scaling of the bound with the number of object copies and is easy to prove via $K^{-1}$, requires more effort to prove if $K^{-1}$ is to be avoided.

Corollary 2.-For a family of density operators that model $N$ independent and identical quantum objects in the form of

$$
\mathbf{F}^{(N)} \equiv\left\{\rho(\theta)^{\otimes N}: \theta \in \Theta \subseteq \mathbb{R}^{p}\right\}
$$

where the tensor power is defined as the tensor product

$$
\rho^{\otimes N} \equiv \underbrace{\rho \otimes \cdots \otimes \rho}_{N \text { terms }}
$$

the efficient influence and the GHB are given by

$$
\delta_{\text {eff }}^{(N)}=\frac{U \delta_{\text {eff }}^{(1)}}{\sqrt{N}}, \quad \tilde{\mathrm{H}}^{(N)}=\frac{\tilde{\mathrm{H}}^{(1)}}{N},
$$

respectively, where $U$ is a map defined as

$$
U h \equiv \frac{1}{\sqrt{N}} \sum_{n=1}^{N} I^{\otimes(n-1)} \otimes h \otimes I^{\otimes(N-n)} .
$$

Proof.-The proof is delegated to Appendix B.

\section{Influence operator via a functional gradient}

Theorem 1 is useful if an influence operator $\delta \in \mathcal{D}$ can be found and $\Pi(\delta \mid \mathcal{T})$ is tractable. One way of deriving an influence operator is to assume that the parameter of interest is a functional $\beta[\rho]$ and consider a derivative of $\beta[\rho]$ in the "direction" of an operator $h$ given by

$$
D_{h} \beta[\rho] \equiv \lim _{\epsilon \rightarrow 0} \frac{\beta[\rho+\epsilon \rho \circ h]-\beta[\rho]}{\epsilon} .
$$

Assume that the directional derivative can be expressed as

$$
D_{h} \beta[\rho]=\operatorname{tr}(\rho \circ h) \tilde{\beta}=\langle h, \tilde{\beta}\rangle \forall h \in \mathcal{Y}
$$

in terms of a $\tilde{\beta} \in \mathcal{Y}$, hereafter called a gradient of $\beta[\rho]$. Any ordinary partial derivative of $\beta$ becomes

$\partial_{j} \beta[\rho]=\lim _{\epsilon \rightarrow 0} \frac{\beta\left[\rho+\epsilon \partial_{j} \rho\right]-\beta[\rho]}{\epsilon}=D_{S_{j}} \beta[\rho]=\left\langle S_{j}, \tilde{\beta}\right\rangle$.

Projecting the gradient into $\mathcal{Z}$ then gives an influence operator, viz.,

$\delta=\Pi(\tilde{\beta} \mid \mathcal{Z})=\tilde{\beta}-\Pi\left(\tilde{\beta} \mid \mathcal{Z}^{\perp}\right)=\tilde{\beta}-\langle\tilde{\beta}, I\rangle \in \mathcal{D}$,

as it is straightforward to check that $\langle\delta, I\rangle=0$ and $\langle S, \delta\rangle=\partial \beta$. The top flowchart in Fig. 2 illustrates the steps to obtain $\delta$ from $\beta[\rho] . \tilde{\beta}, \delta$, and $\delta_{\text {eff }}$ are all gradients that satisfy Eq. (3.34); the difference lies in the set of directions to which each is restricted. $\delta$, for instance, is restricted to $\mathcal{Z}$ and orthogonal to $\mathcal{Z}^{\perp}$, while $\delta_{\text {eff }}$ is restricted to $\mathcal{T}$ and orthogonal to $\mathcal{T}^{\perp}$ [41].

Now consider some examples. The first is $\beta=\operatorname{tr} \rho Y$ for a given (i.e., $\theta$-independent) observable $Y$, which leads to

$$
D_{h} \beta=\operatorname{tr}(\rho \circ h) Y=\langle h, Y\rangle, \quad \delta=Y-\beta .
$$




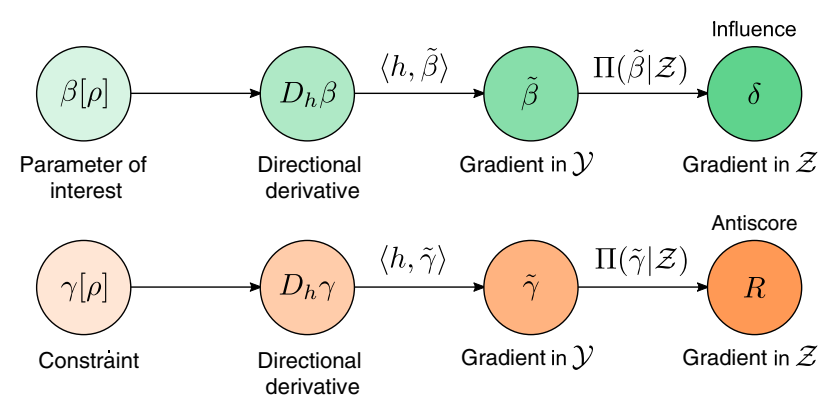

FIG. 2. Top (for Sec. III D): Steps to obtain an influence operator $\delta$ from the functional $\beta[\rho]$ via Eqs. (3.33), (3.34), and (3.36). Bottom (for Sec. VA): Steps to obtain the antiscore operators that span $\mathcal{T}^{\perp}$ via Eqs. (5.2) and (5.3).

The second example is the purity $\beta=\operatorname{tr} \rho^{2}$, which leads to $D_{h} \beta=\operatorname{tr}[(\rho \circ h) \rho+\rho(\rho \circ h)]=\langle h, 2 \rho\rangle, \quad \delta=2(\rho-\beta)$.

The final example is the relative entropy $\beta=\operatorname{tr} \rho(\ln \rho-$ $\ln \sigma)$ [8,42], where $\ln \rho=\sum_{j}\left(\ln \lambda_{j}\right)\left|e_{j}\right\rangle\left\langle e_{j}\right|$ and $\sigma$ is a given density operator with $\operatorname{supp}(\sigma) \supseteq \operatorname{supp}(\rho)$. The differentiability of $\beta$ is not a trivial question when $d=\infty$ [42], but for $d<\infty$ it can be done to give

$$
D_{h} \beta=\langle h, \ln \rho-\ln \sigma\rangle, \quad \delta=\ln \rho-\ln \sigma-\beta,
$$

where $D_{h} \beta$ uses the fact that $\operatorname{tr} \rho[\ln (\rho+\epsilon \rho \circ h)-\ln \rho]$ is second order in $\epsilon$ for any $h \in \mathcal{Z}$ (see Theorem 6.3 in Ref. [8]). The von Neumann entropy is a simple variation of this example.

\section{E. Projection into the tangent space}

The next step is $\Pi(\delta \mid \mathcal{T})$. If the family of density operators is large enough, $\mathcal{T}$ can fill the entire $\mathcal{Z}$, and the projection becomes trivial. We call a family full dimensional if its tangent space at each $\rho$ satisfies

$$
\mathcal{T}=\mathcal{Z}
$$

For a specific example, consider the orthonormal basis of $\mathcal{H}$ given by Eq. (3.2) and the most general parametrization of $\rho$ for $d<\infty$ given by [29]

$\mathbf{F}_{0} \equiv\left\{\rho(\theta)=\sum_{j} \theta_{a j} a_{j}+\sum_{k_{1}<k_{2}}\left(\theta_{b k} b_{k}+\theta_{c k} c_{k}\right)\right\}$,

where

$$
\begin{gathered}
a_{j}=|j\rangle\langle j|, \\
b_{k}=\frac{1}{2}\left(\left|k_{1}\right\rangle\left\langle k_{2}|+| k_{2}\right\rangle\left\langle k_{1}\right|\right), \quad k_{1}<k_{2},
\end{gathered}
$$

$$
c_{k}=\frac{i}{2}\left(\left|k_{1}\right\rangle\left\langle k_{2}|-| k_{2}\right\rangle\left\langle k_{1}\right|\right), \quad k_{1}<k_{2},
$$

and a special entry $\theta_{a 0}$ is removed from the parameters and set as $\theta_{a 0}=1-\sum_{j \neq 0} \theta_{a j}$, such that $\operatorname{tr} \rho(\theta)=\sum_{j} \theta_{a j}=1$ and

$$
p=d^{2}-1 \text {. }
$$

$\partial \rho$ is then given by

$\partial_{a j} \rho=a_{j}-a_{0}, \quad \partial_{b k} \rho=b_{k}, \quad \partial_{c k} \rho=c_{k}$.

The next theorem is a key step in deriving simple analytic results.

Theorem 2.-The $\mathbf{F}_{0}$ family is full dimensional.

Proof.-Consider the solution to $\langle S, h\rangle=0$ for an $h \in \mathcal{Z}$. All operators are bounded if $d<\infty$. We can then use Eqs. (3.18) and (3.46) to obtain

$$
\begin{array}{r}
\operatorname{tr}\left(\partial_{a j} \rho\right) \hat{h}=\langle j|\hat{h}| j\rangle-\langle 0|\hat{h}| 0\rangle=0, \\
\operatorname{tr}\left(\partial_{b k} \rho\right) \hat{h}=\operatorname{Re}\left\langle k_{1}|\hat{h}| k_{2}\right\rangle=0, \quad k_{1}<k_{2}, \\
\operatorname{tr}\left(\partial_{c k} \rho\right) \hat{h}=\operatorname{Im}\left\langle k_{1}|\hat{h}| k_{2}\right\rangle=0, \quad k_{1}<k_{2},
\end{array}
$$

where $\hat{h}$ is any operator in the equivalence class of $h$. Thus, all the diagonal entries of $\hat{h}$ are equal to $\langle 0|\hat{h}| 0\rangle$, and all the off-diagonal entries are zero. In other words, $\hat{h}=\langle 0|h| 0\rangle \hat{I}$, where $\hat{I}$ is the identity operator. But $h \in \mathcal{Z}$ also means that $\operatorname{tr} \rho \hat{h}=\langle 0|\hat{h}| 0\rangle=0$, resulting in $\hat{h}=0$ as the only solution. Hence, $\mathcal{T}^{\perp}=\{0\}$ contains only the zero element, and $\mathcal{T}=\mathcal{Z}$.

$\mathbf{F}_{0}$ implies that the experimenter knows nothing about the density operator, apart from the Hilbert space $\mathcal{H}$ on which it operates. Despite the high dimension of the family, Theorems 1 and 2 turn the problem into a trivial exercise once an influence operator is found, since a $\delta \in \mathcal{D} \subseteq \mathcal{Z}$ is already in $\mathcal{Z}=\mathcal{T}$ and, hence, efficient. Corollary 2 can then be used to extend the result for $N$ copies. For $\beta=\operatorname{tr} \rho Y$, Eq. (3.37) leads to

$$
\tilde{\mathrm{H}}^{(N)}=\frac{\|\delta\|^{2}}{N}=\frac{1}{N} \operatorname{tr} \rho(Y-\beta)^{2} .
$$

This result implies that a von Neumann measurement of $Y$ of each copy and taking the sample mean of the outcomes are already efficient; no other measurement can do better in terms of unbiased estimation. For $\beta=\operatorname{tr} \rho^{2}$, Eq. (3.38) leads to

$$
\tilde{\mathrm{H}}^{(N)}=\frac{\|\delta\|^{2}}{N}=\frac{4}{N} \operatorname{tr} \rho(\rho-\beta)^{2},
$$


and, for $\beta=\operatorname{tr} \rho(\ln \rho-\ln \sigma)$, Eq. (3.39) leads to

$$
\tilde{\mathrm{H}}^{(N)}=\frac{\|\delta\|^{2}}{N}=\frac{1}{N} \operatorname{tr} \rho(\ln \rho-\ln \sigma-\beta)^{2} .
$$

Intriguingly, this expression coincides with the information variance that has found uses in other contexts of quantum information theory, such as quantum hypothesis testing [43].

Deriving Eqs. (3.50)-(3.52) via the conventional bruteforce method would entail the following steps:

(1) Assume the $\mathbf{F}_{0}$ family of density operators given by Eq. (3.41), with $p=d^{2}-1$ parameters.

(2) Compute the $p$ score operators via Eq. (3.4).

(3) Compute the $p$-by- $p$ Helstrom information matrix $K$ via Eq. (3.9).

(4) Compute the inverse $K^{-1}$.

(5) Compute $\beta(\theta)$ via Eq. (3.41), $\partial \beta(\theta)$, and the Helstrom bound via Eq. (3.8).

While this method has been used before to produce Eq. (3.50) [32], it is less clear whether it can easily give Eq. (3.51) or (3.52). Contrast the brute-force method with the proposal here:

(1) Compute the influence operator $\delta$ via a functional derivative of $\beta[\rho]$ according to Sec. III D.

(2) Find the tangent space $\mathcal{T}$ of the density-operator family or the orthocomplement $\mathcal{T}^{\perp}$. For example, Theorem 2 shows that $\mathcal{T}$ is full dimensional for the family of arbitrary density operators, while Sec. V later shows that $\mathcal{T}^{\perp}$ may remain tractable for smaller families.

(3) Compute $\quad \delta_{\text {eff }}=\Pi(\delta \mid \mathcal{T})=\delta-\Pi\left(\delta \mid \mathcal{T}^{\perp}\right) \quad$ and $\tilde{\mathrm{H}}=\left\|\delta_{\text {eff }}\right\|^{2}=\operatorname{tr} \rho \delta_{\text {eff }}^{2}$.

Each step is tractable for all the examples here, regardless of the dimensions.

Equations (3.50)-(3.52) are the quantum bounds promised in Sec. II, although they are merely the simplest examples of what the semiparametric methodology can offer, as Secs. V-VII later show.

\section{PARAMETRIC SUBMODELS}

The proof of Theorem 2 works only in the finitedimensional case $\left(p=d^{2}-1<\infty\right)$. For infinitedimensional problems, the beautiful concept of parametric submodels [19-21,34] offers a more rigorous approach. Let

$$
\mathbf{G} \equiv\{\rho(g): g \in \mathcal{G}\}
$$

be a "mother" density-operator family, where $\mathcal{G}$ may be an infinite-dimensional space. The density operators are still assumed to operate on a common separable Hilbert space $\mathcal{H}$. Denote the true density operator in the family as $\rho$. A parametric submodel $\mathbf{F}^{\sigma}$ is defined as any subset of $\mathbf{G}$ that contains the true $\rho$ and has the parametric form of Eq. (3.1). To wit,

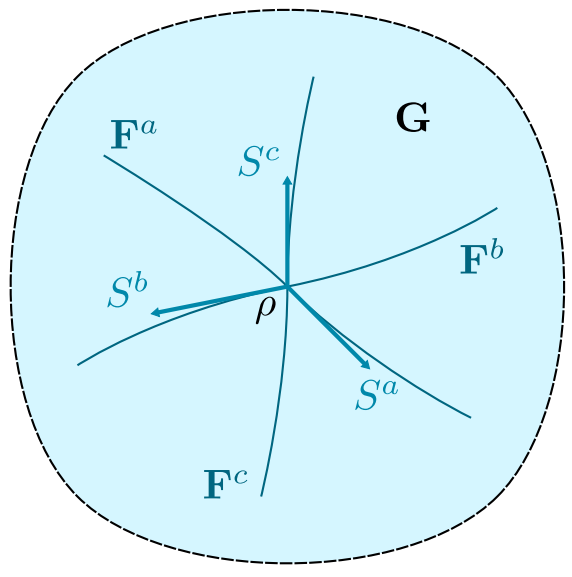

FIG. 3. The space represents $\mathbf{G}$, a mother family of density operators. The true density operator is denoted as $\rho$. Parametric submodels are represented by curves in $\mathbf{G}$ that intersect at $\rho$. Each score $S^{\sigma}$ is a tangent vector that quantifies the "velocity" of a density-operator trajectory in a certain direction.

$$
\mathbf{F}^{\sigma} \equiv\left\{\sigma(\theta): \theta \in \Theta^{\sigma} \subseteq \mathbb{R}^{s}, \sigma(\phi)=\rho\right\} \subseteq \mathbf{G},
$$

where $s$ denotes the dimension of the parameter and $\phi$ denotes the parameter value at which $\sigma(\phi)=\rho$ is the truth; both may be specific to the submodel. In the language of geometry [8,9,27], each $\mathbf{F}^{\sigma}$ is an $s$-dimensional surface in $\mathbf{G}$, and all the surfaces are required to intersect at $\rho$. Figure 3 illustrates the concept.

Each submodel $\mathbf{F}^{\sigma}$ is assumed to be smooth enough for scores to be defined in the same way as before by

$$
(\partial \sigma)_{\theta=\phi}=\rho \circ S^{\sigma},
$$

which denotes a system of $s$ equations given by

$$
\left.\partial_{j} \sigma(\theta)\right|_{\theta=\phi}=\sigma(\phi) \circ S_{j}^{\sigma}(\phi)=\rho \circ S_{j}^{\sigma}(\phi) .
$$

As everything is evaluated at the true $\rho$, the scores of all submodels, in fact, live in the same Hilbert space $\mathcal{Z}$ with respect to $\rho$. Let the set of all parametric submodels of $\mathbf{G}$ with respect to the truth be

$$
\mathcal{F} \equiv\left\{\mathbf{F}^{\sigma}: \sigma \in \mathcal{S}\right\}
$$

where $\mathcal{S}$ denotes the set of indices that label all the submodels. Define the tangent set as the set of the scores from all such parametric submodels of $\mathbf{G}$, viz.,

$$
\{S\} \equiv \bigcup_{\sigma \in \mathcal{S}}\left\{S^{\sigma}\right\}
$$

and the tangent space as the span of the set, viz.,

$$
\mathcal{T} \equiv \overline{\operatorname{span}}\{S\} \subseteq \mathcal{Z} .
$$


An influence operator is now defined as any operator that satisfies the unbiasedness condition for all submodels with respect to $\{S\}$. The condition can be expressed as

$$
\left\langle S^{\sigma}, \delta\right\rangle=(\partial \beta)_{\theta=\phi} \forall \mathbf{F}^{\sigma} \in \mathcal{F},
$$

where $(\partial \beta)_{\theta=\phi}$ is specific to each submodel. If $\langle S, \delta\rangle=\partial \beta$ in Eq. (3.21) is taken to mean Eq. (4.8), then the influenceoperator set $\mathcal{D}$ is still defined by Eq. (3.21). The error operator given by Eq. (3.22) for an unbiased measurement still satisfies Eq. (4.8) by the generic arguments in Sec. 6.2 in Ref. [7], which apply to any submodel, so the error operator remains in $\mathcal{D}$, and Eq. (3.24) still holds. Theorem 1 can now be extended for the mother family.

Theorem 3.- The GHB in Eq. (3.24) for the mother family $\mathbf{G}$ is given by

$$
\tilde{\mathrm{H}}=\min _{\delta \in \mathcal{D}}\|\delta\|^{2}=\left\|\delta_{\mathrm{eff}}\right\|^{2},
$$

where the efficient influence $\delta_{\text {eff }}$ is the unique element in the influence-operator set $\mathcal{D}$ given by

$$
\delta_{\text {eff }}=\Pi(\delta \mid \mathcal{T}),
$$

$\delta$ is any influence operator in $\mathcal{D}$, and $\mathcal{T}$ is the tangent space spanned by the scores of all parametric submodels of $\mathbf{G}$.

Proof.-The proof is identical to that of Theorem 1 if one takes $\{S\}$ to be the tangent set containing the scores of all parametric submodels.

Corollary 2 can also be generalized in an almost identical way, although the proof requires more careful thought.

Corollary 3.-For a family of density operators that model $N$ independent and identical quantum objects in the form of

$$
\mathbf{G}^{(N)} \equiv\left\{\rho(g)^{\otimes N}: g \in \mathcal{G}\right\},
$$

the efficient influence and the GHB are given by

$$
\delta_{\mathrm{eff}}^{(N)}=\frac{U \delta_{\mathrm{eff}}^{(1)}}{\sqrt{N}}, \quad \tilde{\mathrm{H}}^{(N)}=\frac{\tilde{\mathrm{H}}^{(1)}}{N},
$$

respectively, where $\delta_{\mathrm{eff}}^{(1)}$ and $\tilde{\mathrm{H}}^{(1)}$ are those for the $N=1$ family according to Theorem 3 and $U$ is the map given by Eq. (3.32).

Proof.-The proof is delegated to Appendix C.

We now generalize Theorem 2 for infinite-dimensional systems. The following theorem is also a more precise generalization of a classic result in semiparametric theory (Example 1 in Sec. 3.2 in Ref. [20]).

Theorem 4.- $\mathbf{G}_{0}$, defined as the family of arbitrary density operators, is full dimensional.

Proof.-We call a Hilbert-space element in $\mathcal{Y}$ bounded and denote it by $\|h\|_{\text {op }}<\infty$ if its equivalence class contains a bounded operator $\hat{h}$. Denote the set of all bounded elements in $\mathcal{Z}$ as

$$
\mathcal{B} \equiv\left\{h \in \mathcal{Z}:\|h\|_{\text {op }}<\infty\right\}
$$

Take any $h \in \mathcal{B}$ and its bounded operator $\hat{h}$. Construct a scalar-parameter exponential family as $[8,9]$

$$
\sigma(\theta)=\frac{\kappa(\theta)}{\operatorname{tr} \kappa(\theta)}, \quad \kappa(\theta)=\exp (\theta \hat{h} / 2) \rho \exp (\theta \hat{h} / 2),
$$

where $\theta \in \mathbb{R}$ and the truth is at $\sigma(0)=\rho$. As $\hat{h}$ is bounded, $\exp (\theta \hat{h} / 2)$ is bounded and strictly positive. As $\rho$ is nonnegative and unit trace, $\kappa(\theta)$ is non-negative and trace class (see Theorem 2.7.2 in Ref. [7]). Moreover, $\operatorname{tr} \kappa(\theta)$ satisfies the properties

$$
\infty>\operatorname{tr} \kappa(\theta)=\operatorname{tr} \rho \exp (\theta \hat{h})>0,
$$

because $\kappa(\theta)$ is trace class and $\exp (\theta \hat{h})$ is strictly positive. Hence, $\sigma(\theta)$ is a valid density operator at any $\theta$. Since $\mathbf{G}_{0}$ contains arbitrary density operators, $\mathbf{F}^{\sigma}=\{\sigma(\theta): \theta \in$ $\mathbb{R}, \sigma(0)=\rho\}$ is a parametric submodel of $\mathbf{G}_{0}$. It is straightforward to show that

$$
(\partial \sigma)_{\theta=0}=\sigma(0) \circ \hat{h}=\rho \circ \hat{h},
$$

so the score for this model can be taken as $S^{\sigma}=h$.

Define a submodel in the same way for every $h \in \mathcal{B}$, such that all of the $\mathcal{B}$ elements are in the tangent set $\{S\}$, leading to $\mathcal{B} \subseteq\{S\} \subseteq \mathcal{T}$. As $\mathcal{T}$ is closed, the limit points of $\mathcal{B}$ must also be in $\mathcal{T}$, and $\overline{\mathcal{B}} \subseteq \mathcal{T}$, where $\overline{\mathcal{B}}$ is the closure of $\mathcal{B}$. Lemma 2 in Appendix D states that $\mathcal{B}$ is a dense subset of $\mathcal{Z}$, so

$$
\mathcal{Z}=\overline{\mathcal{B}} \subseteq \mathcal{T}
$$

Together with the fact $\mathcal{T} \subseteq \mathcal{Z}$, this result implies $\mathcal{T}=\mathcal{Z}$, and the theorem is proved.

A comparison of the proofs of Theorems 2 and 4 shows how the parametric-submodel concept works. Instead of dealing with one large family such as Eq. (3.41), here, one exploits the freedom offered by $\mathbf{G}_{0}$ to specify many $a d$ hoc and elementary submodels. Each submodel in the proof cannot be simpler - the exponential family is simply a type of geodesics through $\rho$ in density-operator space [8]. In fact, we do not have to use the exponential family, and other families may also be used as long as they fit the purpose of the proof. An enormous number of submodels are introduced, one for each $\mathcal{B}$ element in the proof, leading to an extremely overcomplete tangent set. But that presents no trouble for the geometric approach; only the resultant 


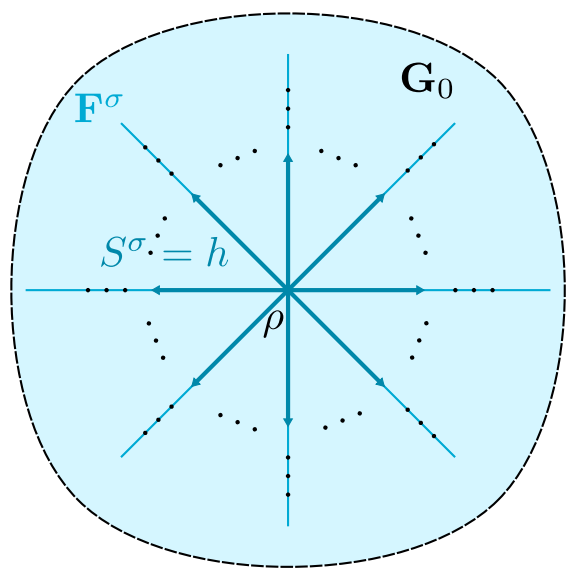

FIG. 4. For any $h \in \mathcal{B}$, one can associate with it an exponential family (a straight line in the density-operator space) that passes through $\rho$. Since $\mathbf{G}_{0}$ contains arbitrary density operators, every line must be contained in $\mathbf{G}_{0}$. It follows that each line is a parametric submodel for $\mathbf{G}_{0}$, and each $h$ should be put in the tangent set. The dots represent the fact that the proof involves lines in all directions and, on each line, scores with all possible norms.

tangent space matters at the end. Figure 4 illustrates the idea.

By virtue of Theorem 4, an influence operator $\delta \in \mathcal{D} \subseteq$ $\mathcal{Z}=\mathcal{T}$ found for a parameter of interest is the efficient one for $\mathbf{G}_{0}$. The examples in Secs. III D and III E work for $\mathbf{G}_{0}$ in the same way they work for $\mathbf{F}_{0}$. If $\beta$ is given by $\beta[\rho]$, an influence operator that satisfies Eq. (4.8) can be found via a gradient of $\beta[\rho]$, as shown in Sec. III D and Fig. 2. In particular, the influence operators given by Eqs. (3.37)-(3.39) and the bounds given by Eqs. (3.50)(3.52) for the various examples should still hold for $\mathbf{G}_{0}$, although the entropy example may require a more rigorous treatment when $d=\infty$ [42].

\section{CONSTRAINED BOUNDS}

\section{A. Antiscore operators}

Consider a constrained family of density operators defined as

$$
\mathbf{G}_{\gamma} \equiv\left\{\rho(g) \in \mathbf{G}_{0}: \gamma[\rho(g)]=0\right\},
$$

where $\gamma[\rho(g)]=0$ denotes a finite set of equality constraints $\left\{\gamma_{k}[\rho(g)]=0: k=1, \ldots, r\right\}$. Such constraints appear often in quantum thermodynamics $[44,45]$. If there exist gradient operators $\left\{\tilde{\gamma}_{k} \in \mathcal{Y}\right\}$ such that, for any $h \in \mathcal{Y}$,

$$
D_{h} \gamma_{k}[\rho]=\left\langle h, \tilde{\gamma}_{k}\right\rangle \text {, }
$$

then each operator given by

$$
R_{k} \equiv \Pi\left(\tilde{\gamma}_{k} \mid \mathcal{Z}\right)=\tilde{\gamma}_{k}-\left\langle\tilde{\gamma}_{k}, I\right\rangle \in \mathcal{Z}
$$

satisfies

$$
D_{h} \gamma_{k}[\rho]=\left\langle h, R_{k}\right\rangle \forall h \in \mathcal{Z},
$$

and the constraint $\gamma[\rho(g)]=0$ implies that $\partial \gamma_{k}[\rho]=$ $\left\langle S^{\sigma}, R_{k}\right\rangle=0$ for all submodels and $k$. In short, we write

$$
\partial \gamma[\rho]=\langle S, R\rangle=0 .
$$

Thus, $\{R\}$ is orthogonal to the tangent set $\{S\}$, and $\operatorname{span}\{R\}$ must be a subset of $\mathcal{T}^{\perp}$. We call $R$ the antiscore operators, as the following theorem shows that they span $\mathcal{T}^{\perp}$ in the same way the scores span $\mathcal{T}$.

Theorem 5.-If $\langle R, R\rangle^{-1}$ exists, $\mathcal{T}^{\perp}=\operatorname{span}\{R\}$ for the $\mathbf{G}_{\gamma}$ family.

Proof.-The proof again follows the classical case (see Example 3 in Sec. 3.2 in Ref. [20]). Let

$\mathcal{R} \equiv \operatorname{span}\{R\}, \quad \mathcal{R}^{\perp} \equiv\{h \in \mathcal{Z}:\langle R, h\rangle=0\}$.

In view of Eq. (5.5),

$$
\mathcal{T} \subseteq \mathcal{R}^{\perp}
$$

Now construct a parametric submodel $\mathbf{F}^{\sigma}$ in terms of each $h \in \mathcal{R}^{\perp}$ as

$\sigma(\theta)=\frac{\kappa(\theta)}{\operatorname{tr} \kappa(\theta)}, \quad \kappa(\theta)=f(\theta h+\theta g) \rho f(\theta h+\theta g)$,

where $\theta \in \mathbb{R}, g=w^{\top} R \in \mathcal{R}$ is an operator to be specified later, and $f(u)$ is defined with respect to the spectral representation of $u=\int \lambda d E_{u}(\lambda)$ as

$$
f(u)=\int\left[1+\tanh \left(\frac{\lambda}{2}\right)\right] d E_{u}(\lambda)
$$

$f(u)$ is bounded and positive even if $u$ is unbounded, so $\sigma(\theta)$ is a valid density operator. Since $\rho \in \mathbf{G}_{\gamma}, \gamma[\rho]=0$. For a $\sigma(\theta)$ away from $\rho$ with $\theta \neq 0$,

$$
\begin{aligned}
\gamma[\sigma(\theta)] & =\gamma[\rho]+\theta D_{h+g} \gamma[\rho]+o(\theta) \\
& =\theta\langle R, h+g\rangle+o(\theta) \\
& =\theta\langle R, g\rangle+o(\theta),
\end{aligned}
$$

where Eq. (5.11) uses Eq. (5.4) and the last step uses the fact $h \in \mathcal{R}^{\perp}$. To make $\sigma(\theta)$ satisfy the constraint $\gamma[\sigma(\theta)]=0, g(\theta)=w(\theta)^{\top} R$ can be set as a function of $\theta$ to cancel the $o(\theta)$ term, with 


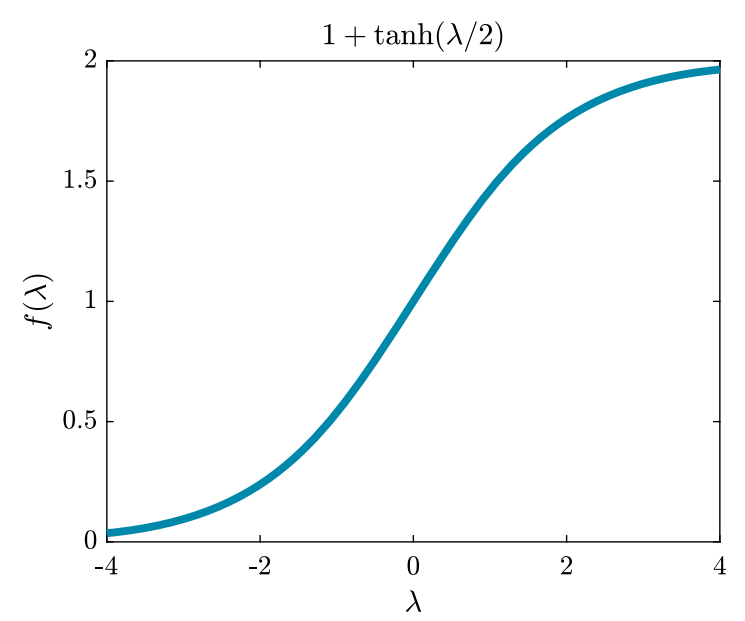

FIG. 5. A plot of $f(\lambda)=1+\tanh (\lambda / 2)$ to illustrate its boundedness and positivity.

$$
w(\theta)=-\langle R, R\rangle^{-1} o(\theta) / \theta .
$$

Then, $\gamma[\sigma(\theta)]=0$ and $\mathbf{F}^{\sigma}$ is a valid parametric submodel of $\mathbf{G}_{\gamma}$. Equation (5.13) also implies that $\theta g(\theta)=o(\theta)$ is negligible relative to $\theta h$ for infinitesimal $\theta$, so the score for $\mathbf{F}^{\sigma}$ is $h$, which should be put in the tangent set $\{S\}$. As this procedure can be done for any $h \in \mathcal{R}^{\perp}$, $\mathcal{R}^{\perp} \subseteq\{S\} \subseteq \mathcal{T}$. Together with Eq. (5.7), this result leads to $\mathcal{T}=\mathcal{R}^{\perp}$, giving $\mathcal{T}^{\perp}=\mathcal{R}$.

The family given by Eqs. (5.8) and (5.9) is more convenient to use here than the exponential family used in the proof of Theorem 4. The $f(u)$ defined by Eq. (5.9) is a generalization of the classical version in Example 1 in Sec. 3.2 in Ref. [20] and plotted in Fig. 5. It is designed to give a valid density operator via Eqs. (5.8) —even if the argument is an unbounded operator-yet produce the desired score when linearized at $\theta=0$. An adjustable operator $g(\theta)$ is included in the submodel to make $\sigma(\theta)$ satisfy the constraint away from $\rho$. Figure 6 further illustrates the idea of the proof.

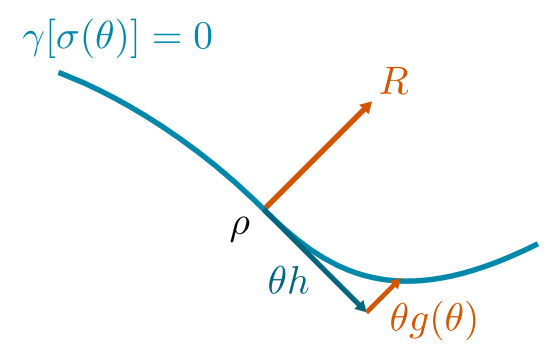

FIG. 6. Each $R$ is a vector normal to the surface defined by $\gamma[\rho(g)]=0$ in density-operator space. For any $h \in \mathcal{R}^{\perp}$, a parametric submodel $\sigma(\theta)$ can be constructed to satisfy the constraint $\gamma[\sigma(\theta)]=0$. Away from $\rho$, a correction $\theta g(\theta)=o(\theta)$ in $\mathcal{R}$ is needed to make $\sigma(\theta)$ stay with the constraint. The tangent vector of the submodel at $\rho$ is still $h$, since the correction is higher order in $\theta$.
Given an influence operator $\delta$, such as those derived in Sec. III D, the efficient influence and the GHB can be computed in terms of $\mathcal{T}^{\perp}$ instead of $\mathcal{T}$ via

$$
\begin{aligned}
& \delta_{\text {eff }}=\Pi(\delta \mid \mathcal{T})=\delta-\Pi\left(\delta \mid \mathcal{T}^{\perp}\right), \\
& \left\|\delta_{\text {eff }}\right\|^{2}=\|\delta\|^{2}-\left\|\Pi\left(\delta \mid \mathcal{T}^{\perp}\right)\right\|^{2} .
\end{aligned}
$$

The same projection formula that gives $\delta_{\text {eff }}$ in Appendix A can be adapted to give

$$
\begin{gathered}
\Pi\left(\delta \mid \mathcal{T}^{\perp}\right)=\langle R, \delta\rangle^{\top}\langle R, R\rangle^{-1} R, \\
\left\|\Pi\left(\delta \mid \mathcal{T}^{\perp}\right)\right\|^{2}=\langle R, \delta\rangle^{\top}\langle R, R\rangle^{-1}\langle R, \delta\rangle .
\end{gathered}
$$

Equations (5.16) and (5.17) remain tractable if the constraints are few. The gradients of $\gamma[\rho]$ can be derived in the same way as those of $\beta[\rho]$, as shown in Fig. 2, and $R$ can be computed analytically for linear constraints, the purity constraint, and the entropy constraint by following the same type of calculations shown in Eqs. (3.37)-(3.39). Equation (2.4) is a special example of the constrained GHB when $\beta=\operatorname{tr} \rho Y$ and $\gamma=\operatorname{tr} \rho(Z-\zeta)=0$.

\section{B. Entropy estimation in quantum thermodynamics}

In quantum thermodynamics, conserved quantities of a dynamical system, such as the energy and the particle number, are expressed as moment constraints on the density operator with respect to a vector of observables $Z$ and a vector of constants $\zeta$, viz.,

$$
\operatorname{tr} \rho Z_{k}=\zeta_{k}, \quad k=1, \ldots, r .
$$

Given such constraints, the density operator is often assumed to be the one with the maximum entropy [44], known as the generalized Gibbs ensemble [45]. Such an assumption, however, requires verification and does not hold out of equilibrium. Experiments on Bose gases have been performed to study the quantum states at different times and the validity of the maximum-entropy principle at steady state [46-48].

When the maximum-entropy principle is in question for those experiments, it is prudent to make no prior assumption about the density operator other than the constraints. Thus, one should consider a family of density operators given by Eq. (5.1), where the vectoral constraint is $\gamma[\rho]=\operatorname{tr} \rho(Z-\zeta)=0$. Suppose that the von Neumann entropy $\beta=-\operatorname{tr} \rho \ln \rho$ is the parameter of interest. The estimation of $\beta$ is then a problem of quantum semiparametric estimation.

As the experiments typically involve high-dimensional systems, quantum state tomography is impractical. A more efficient estimation of $\beta$ should exist. The formalism here leads to a quantum limit given by 


$$
\begin{gathered}
\mathrm{E} \geq \frac{1}{N}\left(\|\delta\|^{2}-\langle R, \delta\rangle^{\top}\langle R, R\rangle^{-1}\langle R, \delta\rangle\right), \\
\delta=-\ln \rho-\beta, \quad R=Z-\zeta .
\end{gathered}
$$

This bound is equivalent to the Holevo bound, as shown in Sec. VIII, so it is asymptotically attainable in principle, at least for finite-dimensional systems [28-30], although the experimental implementation of efficient measurements remains an open question.

As entropy is an excellent measure of randomness and a central quantity in information theory, entropy estimation has many applications beyond thermodynamics. In classical statistics, the semiparametric estimation of entropic quantities is a well-studied problem with known near-efficient estimators and applications in universal coding, statistical tests, random-number generation, econometrics, spectroscopy, and even neuroscience [49]. In the quantum domain, one application is universal quantuminformation compression [50]: Knowing just the von Neumann entropy and nothing else about $\rho$ allows the quantum information to be compressed in accordance with the entropy. Another application is the estimation of an entropic measure of entanglement, which allows one to demonstrate entanglement without full tomography [16]. The quantum limit here quantifies the minimum amount of resources needed to achieve a desired precision. Its asymptotic attainability suggests that it is a lofty but fair yardstick for experimental design.

\section{Philosophy}

The proposed approach to quantum semiparametric bounds is the polar opposite of the usual approach in quantum metrology. In the usual bottom-up approach, one assumes a small family of density operators with a few parameters and computes $\|\Pi(\delta \mid \mathcal{T})\|^{2}$ that is determined by the overlap between $\delta$ and the scores $S$. Here, one starts with a large family with almost full dimension, computes $\|\delta\|^{2}$ for an amenable $\delta$, and then reduces it by $\left\|\Pi\left(\delta \mid \mathcal{T}^{\perp}\right)\right\|^{2}$ that is determined by the overlap between $\delta$ and the antiscores $R$, as illustrated by Fig. 7. The complexity of the problem, thus, depends on the dimension of the family, and the essential insight of this work is that the problem can become simple again when the dimension is close to being full. Of course, if the dimension of $\mathcal{T}^{\perp}$ is high, the topdown approach may also suffer from the curse of dimensionality. The medium families with both $\mathcal{T}$ and $\mathcal{T}^{\perp}$ in high dimensions are the most difficult to deal with, as they may be impregnable from either end.

\section{Looser bounds}

It may often be the case that, despite one's best efforts, the exact $\delta_{\text {eff }}$ for a problem remains intractable. Then, a standard strategy in statistics and quantum metrology is to

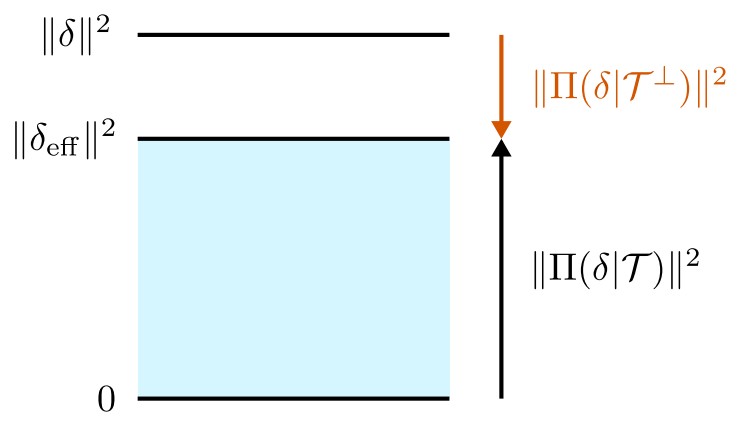

FIG. 7. An illustration of the conventional bottom-up approach to quantum bounds and the top-down approach to semiparametric bounds, as discussed in Sec. V C.

sandwich $\left\|\delta_{\text {eff }}\right\|^{2}$ between upper and lower bounds. $\|\delta\|^{2}$ is an obvious upper bound and can be obtained from the gradient method in Sec. III D if $\beta$ can be expressed as a functional $\beta[\rho]$. Another way is to use Eq. (3.23) if an unbiased measurement and its error are known. The evaluation of lower bounds, on the other hand, can be facilitated by the following proposition.

Proposition 1.-Let $\mathcal{V} \subseteq \mathcal{T}$ be a closed subspace of $\mathcal{T}$ and $\mathcal{V}^{\perp}$ be the orthocomplement of $\mathcal{V}$ in $\mathcal{Z}$. Then

$\tilde{\mathrm{H}}=\left\|\delta_{\mathrm{eff}}\right\|^{2} \geq\|\Pi(\delta \mid \mathcal{V})\|^{2}=\|\delta\|^{2}-\left\|\Pi\left(\delta \mid \mathcal{V}^{\perp}\right)\right\|^{2}$.

In particular, if

$$
\mathcal{V}=\overline{\operatorname{span}}\left\{S^{\sigma}\right\}
$$

is taken as the tangent space for a particular parametric submodel $\mathbf{F}^{\sigma}$, then

$$
\|\Pi(\delta \mid \mathcal{V})\|^{2}=\tilde{\mathrm{H}}^{\sigma}
$$

is the GHB for that submodel.

Proof.-The proof is delegated to Appendix E.

A tight lower bound on $\left\|\delta_{\text {eff }}\right\|^{2}$ can be sought by devising a submodel that is as unfavorable to the estimation of $\beta$ as possible. Another approach is to devise an overconstrained model with $\mathcal{V}^{\perp} \supseteq \mathcal{T}^{\perp}$ and evaluate a lower bound on $\left\|\delta_{\text {eff }}\right\|^{2}$ from the top by overshooting, as illustrated by Fig. 8 .

\section{EXAMPLES IN OPTICS}

\section{A. Quadrature estimation}

Here, we further illustrate the theory with examples in optics, where quantum measurement theory has found the most experimental success [51]. For the first and simplest example, let $\rho$ be a density operator of an optical mode and assume the $\mathbf{G}_{0}$ family of arbitrary density operators. Consider the estimation of the mean of a quadrature operator $Y$, with $\beta=\operatorname{tr} \rho Y$. This problem appears often in optical state characterization, communication, 


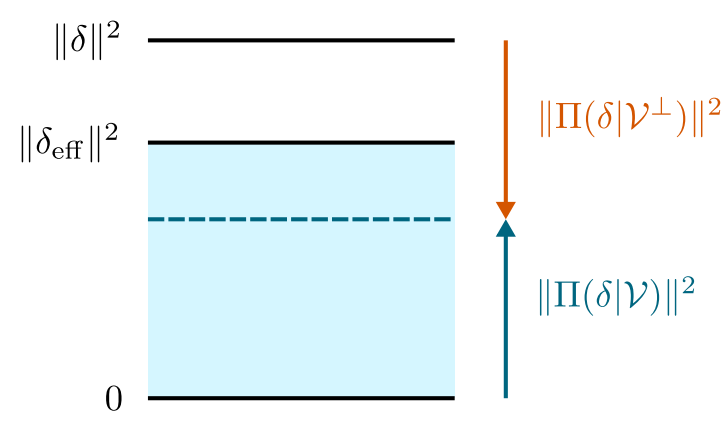

FIG. 8. One can obtain a lower bound on $\left\|\delta_{\text {eff }}\right\|^{2}$ by either undershooting from the bottom via a more amenable subspace $\mathcal{V} \subseteq \mathcal{T}$ or overshooting from the top via an overconstrained model with $\mathcal{V}^{\perp} \supseteq \mathcal{T}^{\perp}$.

and sensing, where $\beta$ is a displacement parameter [17]. The GHB is given by Eq. (3.50), and homodyne detection of $Y$ is efficient. Note that this example is different from all previous studies of quadrature estimation [1,7], which assume Gaussian states or similarly low-dimensional parametric models. The semiparametric scenario here allows $\rho$ to be arbitrary and possibly non-Gaussian.

Now suppose that side information $\operatorname{tr} \rho Z=\zeta$ concerning another quadrature $Z$ is available. It follows from Sec. V that the efficient influence is now

$$
\delta_{\text {eff }}=Y-\beta-\frac{C_{Y Z}}{V_{Z}}(Z-\zeta),
$$

where $C_{Y Z}$ and $V_{Z}$ are given by Eqs. (2.5). The GHB is then given by Eq. (2.4), which is lowered by any correlation between $Y$ and $Z$. From the efficient influence, one may use Eq. (3.22) to find an efficient measurement, which obeys

$$
\int \check{\beta}(\lambda) d E(\lambda)=Y-\frac{C_{Y Z}}{V_{Z}}(Z-\zeta) .
$$

This equation can be satisfied if the POVM measures the quadrature $Y-\left(C_{Y Z} / V_{Z}\right) Z$ instead of the obvious $Y$. Notice, however, that $C_{Y Z} / V_{Z}$ depends on the unknown $\rho$. Whether adaptive measurements [51] can implement this POVM approximately and whether asymptotic attainability is possible for this infinite-dimensional problem are interesting open questions. One approach may be to form rough estimates of the covariances $C_{Y Z}$ and $V_{Z}$ via heterodyne detection of a portion of the light first and then measure the desired quadrature via homodyne detection based on the approximate $C_{Y Z} / V_{Z}$.

\section{B. Family of classical states}

For a more nontrivial example, consider a densityoperator family in the form

$$
\mathbf{G}_{c} \equiv\left\{\rho(P)=\int d^{2} \alpha P(\alpha)|\alpha\rangle\langle\alpha|: P \in \mathcal{G}\right\},
$$

$$
\mathcal{G}=\text { all positive probability densities, }
$$

where $\alpha=\alpha^{\prime}+i \alpha^{\prime \prime} \in \mathbb{C}, d^{2} \alpha=d \alpha^{\prime} d \alpha^{\prime \prime},|\alpha\rangle$ is a coherent state, and $P$ is the Glauber-Sudarshan function [52]. As $P$ is assumed to be positive, $\mathbf{G}_{c}$ is a family of classical states [52] and a strict subset of $\mathbf{G}_{0}$. The assumption of $\mathbf{G}_{c}$ instead of $\mathbf{G}_{0}$ is more appropriate for practical applications with significant decoherence, as nonclassical states are unlikely to survive in such an environment.

Consider a moment parameter of the form

$$
\beta(P)=\int d^{2} \alpha P(\alpha) f\left(\alpha, \alpha^{*}\right)
$$

where $f\left(\alpha, \alpha^{*}\right)$ is a real polynomial of $\alpha$ and $\alpha^{*}$. For example, one may be interested in the mean of a quadrature, in which case $f=\alpha \exp (-i \theta)+\alpha^{*} \exp (i \theta)$, or the mean energy, in which case $f=|\alpha|^{2}$. The optical equivalence theorem [52] gives

$$
\beta=\operatorname{tr} \rho Y, \quad Y=: f\left(a, a^{\dagger}\right):,
$$

where : $f\left(a, a^{\dagger}\right)$ : denotes the normal ordering [52]. It follows from Sec. III D that an influence operator is $\delta=Y-\beta$.

The next step is to find the tangent space of $\mathbf{G}_{c}$. Although $\mathbf{G}_{c}$ is a smaller family than $\mathbf{G}_{0}$, its dimension turns out to be just as high.

Proposition 2. $-\mathbf{G}_{c}$ is full dimensional.

Proof.-The proof is delegated to Appendix F.

With the full-dimensional tangent space, the GHB is also given by Eq. (3.50). This result shows that the obvious von Neumann measurement of $Y$ remains efficient in estimating $\beta$, and no alternative measurements can do better, despite restricting the family to classical states. For example, if $f\left(\alpha, \alpha^{*}\right)$ is a quadrature, then the homodyne measurement is efficient, and if $f\left(\alpha, \alpha^{*}\right)=|\alpha|^{2}$, then $: f\left(a, a^{\dagger}\right):=a^{\dagger} a$, and the photon-number measurement is efficient.

$\mathcal{G}$, the space of positive densities, is infinite dimensional. The estimation of $P$ is a nonparametric problem [53], in contrast with the semiparametric problems studied here. In classical statistics, it is known that a nonparametric estimation of the probability density cannot achieve a parametric convergence rate $[\mathrm{E}=O(1 / N)][19,20,54]$, and this difficulty is expected to translate to the quantum domain. Semiparametric estimation, on the other hand, can achieve the parametric rate and is the more feasible task if one is interested in only a few parameters of the system.

A further restriction on the family of $P$ can give very different results, as shown in the next section in the context of incoherent imaging. 


\section{Incoherent imaging}

\section{The mother model}

Here, we summarize existing results concerning the problem of incoherent imaging [5] using the language of semiparametrics. Unlike previous sections, this section presents no new results essentially. Rather, the goal is to use this very important but equally difficult problem to illustrate the concepts and current limitations of the quantum semiparametric theory.

The basic setup of an imaging system is depicted in Fig. 9. The object is assumed to emit spatially incoherent light at an optical frequency. For simplicity, the imaging system is assumed to be one dimensional, paraxial, and diffraction limited. A model of each photon on the image plane is $[5,15,55]$

$$
\begin{gathered}
\mathbf{G}=\left\{\rho(F)=\int d X F(X)\left|\psi_{X}\right\rangle\left\langle\psi_{X}\right|: F \in \mathcal{G}_{1}\right\}, \\
\left|\psi_{X}\right\rangle=\int d x \psi(x-X)|x\rangle=\exp (-i k X)\left|\psi_{0}\right\rangle,
\end{gathered}
$$

where $F$ is the unknown source density, $\mathcal{G}_{1}$ is a set of probability densities on $\mathbb{R}, X \in \mathbb{R}$ is the object-plane coordinate, $\psi(x)$ is the point-spread function of the imaging system, $x \in \mathbb{R}$ is the image-plane coordinate normalized with respect to the magnification factor [56], $|x\rangle$ is the Dirac position ket that satisfies $\left\langle x \mid x^{\prime}\right\rangle=\delta\left(x-x^{\prime}\right)$, and $k$ is the canonical momentum operator. $X$ and $x$ are further assumed to be normalized with respect to the width of $\psi(x)$ so that they are dimensionless. $\psi(x)$ is assumed here to be

$$
\psi(x)=\frac{1}{(2 \pi)^{1 / 4}} \exp \left(-\frac{x^{2}}{4}\right),
$$

such that $\left|\psi_{X}\right\rangle=|\alpha=X / 2\rangle$ is a coherent state. Various generalizations can be found in Refs. [5,15,24,55], and references therein. Besides imaging, the model can also be used to describe a quantum particle under random displacements [13,57].

The problem is semiparametric if $\mathcal{G}_{1}$ is infinite dimensional, such as

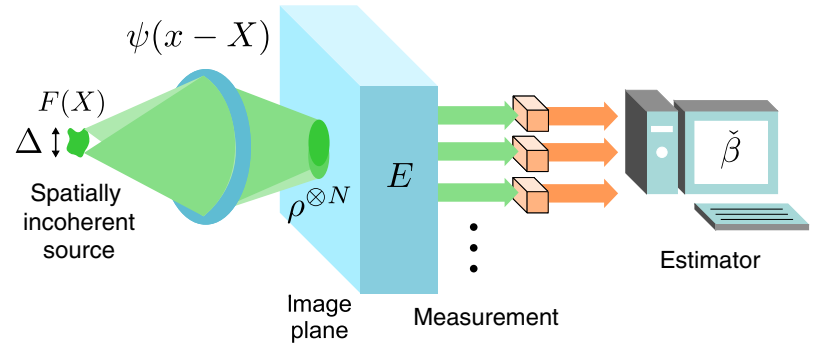

FIG. 9. A far-field incoherent optical imaging system.

$$
\mathcal{G}_{1}=\text { all probability densities on } \mathbb{R},
$$

and the parameter of interest is a functional of $F$, such as the object moment

$$
\beta_{\mu}(F)=\int d X F(X) X^{\mu}
$$

where $\mu \in \mathbb{N}_{1}$ denotes the order of the moment of interest. Notice that the family indicated by Eq. (6.10) is much smaller than the one given by Eq. (6.4) in the previous example, as the Glauber-Sudarshan function is now separable in terms of $\left(\alpha^{\prime}, \alpha^{\prime \prime}\right)$ and confined to the real axis of $\alpha$, viz.,

$$
P(\alpha)=2 F\left(2 \alpha^{\prime}\right) \delta\left(\alpha^{\prime \prime}\right)
$$

In fact, the dimension of $\mathcal{T}^{\perp}$ is now infinite, as shown in Appendix G, so this problem is the most difficult type described in Sec. V C.

The errors and their bounds are all functionals of the true density $F$, and we focus on their values for subdiffraction distributions, which are defined as those with a width $\Delta$ around $X=0$ much smaller than the point-spread-function width or, in other words, $\Delta \ll 1[5]$.

\section{Semiparametric measurements and estimators}

Two globally unbiased measurements for semiparametric moment estimation are known [24]. For $N$ detected photons [58], both are separable measurements and sample means in the form of [59]

$$
\begin{array}{cc}
E^{(N)}\left(\mathcal{A}_{1}, \mathcal{A}_{1}, \ldots, \mathcal{A}_{N}\right)=\bigotimes_{n=1}^{N} E\left(\mathcal{A}_{n}\right), & \mathcal{A}_{n} \in \Sigma_{\mathcal{X}}, \\
\check{\beta}^{(N)}\left(\lambda_{1}, \lambda_{2}, \ldots, \lambda_{N}\right)=\frac{1}{N} \sum_{n=1}^{N} \check{\beta}\left(\lambda_{n}\right), & \lambda_{n} \in \mathcal{X} .
\end{array}
$$

The first measurement is direct imaging, which measures the intensity on the image plane and is equivalent to the projection of each photon in the position basis as

$$
d E^{\text {(direct) }}(x)=d x|x\rangle\langle x|, \quad x \in \mathcal{X}=\mathbb{R} .
$$

An unbiased semiparametric estimator is given by the sample mean of

$$
\begin{gathered}
\check{\beta}_{\mu}^{\text {(direct) }}(x)=\sum_{\nu=0}^{\mu}\left(C^{-1}\right)_{\mu \nu} x^{\nu}, \\
C_{\mu \nu}=1_{\mu \geq \nu}\left(\begin{array}{l}
\mu \\
\nu
\end{array}\right) \int d x|\psi(x)|^{2} x^{\mu-\nu},
\end{gathered}
$$




$$
1_{\text {proposition }}= \begin{cases}1, & \text { if proposition is true } \\ 0, & \text { otherwise }\end{cases}
$$

and the error is

$$
\mathrm{E}_{\mu}^{(\text {direct })}=\frac{O(1)}{N},
$$

where $O(1)$ denotes a prefactor that does not scale with $\Delta$ in the first order. The second measurement is the so-called spatial-mode demultiplexing (SPADE) [4,5,15,24,55], which demultiplexes the image-plane light in the Hermite-Gaussian basis given by

$$
\begin{gathered}
\left|\phi_{m}\right\rangle=\int d x \phi_{m}(x)|x\rangle, \quad m \in \mathbb{N}_{0}, \\
\phi_{m}(x)=\frac{\mathrm{He}_{m}(x)}{(2 \pi)^{1 / 4} \sqrt{m !}} \exp \left(-\frac{x^{2}}{4}\right)
\end{gathered}
$$

where $\operatorname{He}_{m}(x)$ is a Hermite polynomial [60]. For the estimation of an even moment with $\mu=2 j$, the POVM for each photon is

$$
E^{(\mathrm{SPADE})}(m)=\left|\phi_{m}\right\rangle\left\langle\phi_{m}\right|, \quad m \in \mathcal{X}=\mathbb{N}_{0},
$$

an unbiased semiparametric estimator is given by the sample mean of

$$
\check{\beta}_{2 j}^{(\mathrm{SPADE})}(m)=1_{m \geq j} \frac{4^{j} m !}{(m-j) !},
$$

and the error is

$$
\mathrm{E}_{2 j}^{(\mathrm{SPADE})}=\frac{O\left(\Delta^{2 j}\right)}{N}=\frac{O\left(\Delta^{\mu}\right)}{N},
$$

which is much lower than that of direct imaging in the subdiffraction regime for the second and higher moments. For the estimation of odd moments with SPADE, only approximate results have been obtained so far $[14,55,61,62]$ and are not elaborated here.

Both estimators are efficient for their respective measurements in the classical sense [24]. In the quantum case, the question is whether SPADE is efficient or there exist even better measurements. Computing the GHB, or at least bounding it, would answer the question and establish the fundamental quantum efficiency for incoherent imaging.

\section{Lower bounds via parametric submodels}

Both Eqs. (6.19) and (6.24) are upper bounds on the GHB. By virtue of Proposition 1, all earlier quantum lower bounds derived for incoherent imaging via parametric models are, in fact, lower bounds on the GHB for the mother family given by Eq. (6.7), with the true $\rho$ being evaluated at certain special cases of $F$. References [4,63], for example, assume discrete point sources, but exact results become difficult to obtain for a large number of sources. Here, we highlight two methods that work for any $F$ but can give only looser bounds.

The first method is the culmination of Sec. 6 in Ref. [55] and Appendix C in Ref. [15]. Assume that

$$
\theta=\left(\begin{array}{c}
\theta_{g} \\
\theta_{h}
\end{array}\right)
$$

consists of two sets of parameters $\theta_{g}=\left(\theta_{g 1}, \theta_{g 2}, \ldots\right)^{\top}$ and $\theta_{h}=\left(\theta_{h 0}, \theta_{h 1}, \ldots\right)^{\top}$. Define a submodel given by

$$
\begin{array}{r}
\sigma(\theta)=\int d X F(X \mid \theta)\left|\psi_{X}\right\rangle\left\langle\psi_{X}\right|, \\
F(X \mid \theta)=\int d Y \delta\left(X-h\left(Y \mid \theta_{h}\right)\right) G\left(Y \mid \theta_{g}\right), \\
G\left(Y \mid \theta_{g}\right)=\frac{\left[1+\tanh g\left(Y \mid \theta_{g}\right)\right] F(Y)}{\int d Y\left[1+\tanh g\left(Y \mid \theta_{g}\right)\right] F(Y)} .
\end{array}
$$

The truth is at

$$
\begin{gathered}
\sigma(0)=\rho, \quad F(X \mid 0)=G(X \mid 0)=F(X), \\
h(Y \mid 0)=Y, \quad g(Y \mid 0)=0 .
\end{gathered}
$$

$\sigma(\theta)$ can be rewritten as

$$
\sigma(\theta)=\int d Y G\left(Y \mid \theta_{g}\right)\left|\psi_{h\left(Y \mid \theta_{h}\right)}\right\rangle\left\langle\psi_{h\left(Y \mid \theta_{h}\right)}\right| .
$$

In other words, we introduce parameters to both the mixing density and the displacement in the model by rewriting the mixture. Appendix $\mathrm{H}$ shows how the extended convexity of the Helstrom information $[13,64]$ can be used on Eq. (6.31) to give

$$
\tilde{\mathrm{H}}_{\mu}^{(N)} \geq \frac{\mathrm{H}_{\mu}^{\sigma}}{N} \geq \frac{\beta_{2 \mu}-\beta_{\mu}^{2}+\mu^{2} \beta_{2 \mu-2}}{N}=\frac{O\left(\Delta^{2 \mu-2}\right)}{N} .
$$

A more careful calculation shows that the SPADE error is exactly equal to this bound for $\mu=2$ [24]. For higher moments, however, Eq. (6.32) remains much lower than that achievable by SPADE.

The second method, as reported in Ref. [15], considers the formal expansion $\exp (-i k X)=\sum_{p=0}^{\infty}(-i k X)^{p} / p$ !, which leads to

$$
\sigma(\theta)=\sum_{p_{1}=0}^{\infty} \sum_{p_{2}=0}^{\infty} \beta_{p_{1}+p_{2}} \frac{(-i k)^{p_{1}}}{p_{1} !}\left|\psi_{0}\right\rangle\left\langle\psi_{0}\right| \frac{(i k)^{p_{2}}}{p_{2} !} .
$$


Consider this expression as a parametric submodel with only one scalar parameter $\theta=\beta_{\mu}$ for a given $\mu$, while all the other moments $\beta_{\nu}$ with $\nu \neq \mu$ are fixed. Then, the Helstrom bound for this submodel is simply $\mathrm{H}_{\mu}^{\sigma}=1 / K_{\mu \mu}^{\sigma}$, where $K_{\mu \mu}^{\sigma}$ is the Helstrom information with respect to $\theta=\beta_{\mu}$. Reference [15] finds via a purification technique that this Helstrom bound is, in turn, bounded by

$$
\mathrm{H}_{\mu}^{\sigma}=\frac{1}{K_{\mu \mu}^{\sigma}} \geq O\left(\Delta^{2\lfloor\mu / 2\rfloor}\right) .
$$

By virtue of Corollary 3 and Proposition 1, we obtain

$$
\tilde{\mathrm{H}}_{\mu}^{(N)} \geq \frac{\mathrm{H}_{\mu}^{\sigma}}{N} \geq \frac{O\left(\Delta^{2\lfloor\mu / 2\rfloor}\right)}{N} .
$$

This lower bound does match the performance of SPADE in order of magnitude, but it does not have a simple closedform expression, and the question of whether SPADE is exactly efficient for moments higher than the second remains open.

\section{SEMIPARAMETRIC ESTIMATION WITH EXPLICIT NUISANCE PARAMETERS}

\section{A. The efficient score operator}

We now consider problems where there is an explicit partition of the parameters into a scalar $\beta$ and nuisance parameters $\eta$ that may be infinite dimensional, viz.,

$$
\mathbf{G}=\left\{\rho(\beta, \eta): \beta \in \Theta_{\beta} \subseteq \mathbb{R}, \eta \in \mathcal{G}\right\} .
$$

An example is the displacement model given by Eq. (2.6), where $\beta$ is the displacement parameter and the initial state $\rho_{0}$ depends on the nuisance parameters. All previous studies of the problem assume that $\rho_{0}$ is known exactly. In practice, however, $\rho_{0}$ may be poorly characterized, and the estimation performance in the presence of unknown nuisance parameters may suffer as a result.

With the explicit partition of the parameters, the scores can be partitioned similarly. Let $S^{\beta}$ be the score with respect to the parameter of interest, as defined by

$$
\frac{\partial \rho(\beta, \eta)}{\partial \beta}=\rho \circ S^{\beta},
$$

where $\eta$ is fixed at the truth. To define the nuisance scores, consider the subfamily

$$
\mathbf{G}_{\eta} \equiv\{\rho(\beta, \eta): \eta \in \mathcal{G}\},
$$

which holds $\beta$ fixed at the truth instead. Define the nuisance tangent set $\left\{S^{\eta}\right\}$ as the set of scores from all parametric submodels of $\mathbf{G}_{\eta}$ and the nuisance tangent space as

$$
\Lambda \equiv \overline{\operatorname{span}}\left\{S^{\eta}\right\}
$$

The unbiasedness condition for an influence operator becomes

$$
\left\langle S^{\beta}, \delta\right\rangle=\frac{\partial \beta}{\partial \beta}=1, \quad\left\langle S^{\eta}, \delta\right\rangle=0 .
$$

The second of Eqs. (7.5) implies that $\delta \perp \Lambda$, so if $S^{\beta} \in \Lambda$, $\left\langle S^{\beta}, \delta\right\rangle=0$, and no influence operator that obeys both Eqs. (7.5) can exist. In that case, we assume the GHB to be infinite. Provided that $S^{\beta} \notin \Lambda$, however, the following theorem provides another method of computing the efficient influence and the GHB.

Theorem 6.-Assuming $S^{\beta} \notin \Lambda$ and the unbiasedness condition given by Eqs. (7.5), the efficient influence and the GHB are given by

$$
\delta_{\text {eff }}=\frac{S_{\text {eff }}}{\left\|S_{\text {eff }}\right\|^{2}}, \quad \tilde{\mathrm{H}}=\frac{1}{\left\|S_{\text {eff }}\right\|^{2}},
$$

respectively, where $S_{\text {eff }}$, henceforth called the efficient score, is given by

$$
S_{\text {eff }}=S^{\beta}-\Pi\left(S^{\beta} \mid \Lambda\right) .
$$

Proof.-The proof is delegated to Appendix I.

Figure 10 illustrates the Hilbert-space concepts involved in Theorem 6. We note that Sec. V in Ref. [36] also arrives at conclusions similar to Theorem 6 in the parametric case, but the crucial point here is the Hilbert-space approach, which enables us to derive closed-form solutions to semiparametric problems, as shown in the next section.

\section{B. Displacement estimation with a constrained family of initial states}

Consider the displacement model given by Eq. (2.6) and illustrated by Fig. 11. For high-dimensional systems, only a

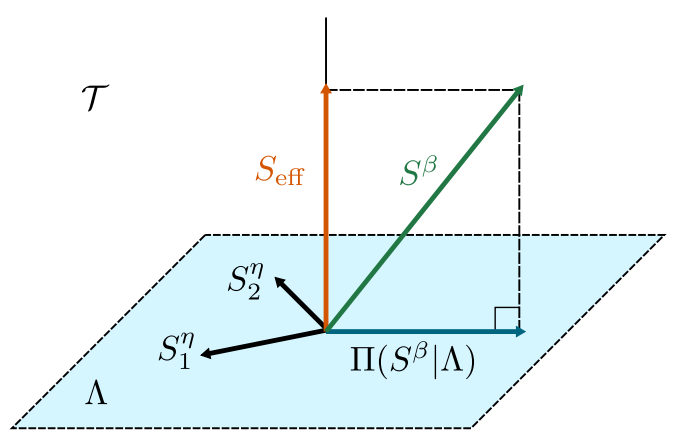

FIG. 10. The whole space in the picture represents the tangent space $\mathcal{T}$. $\Lambda$ is the nuisance tangent space spanned by the nuisance tangent set $\left\{S^{\eta}\right\} . S^{\beta}$ is the score with respect to the parameter of interest. The efficient score $S_{\text {eff }}$ is $S^{\beta}$ minus its projection $\Pi\left(S^{\beta} \mid \Lambda\right)$. The result is orthogonal to $\Lambda$. 


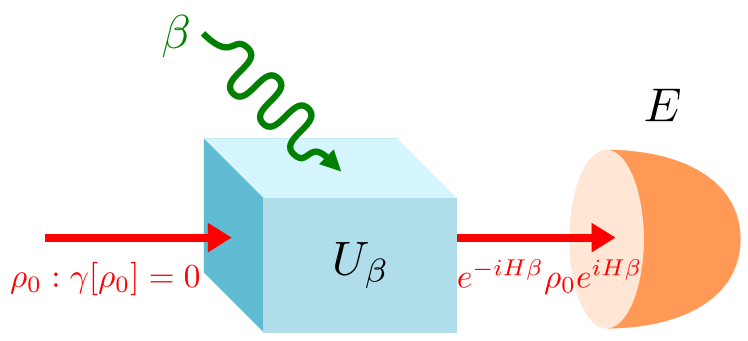

FIG. 11. A schematic of the semiparametric displacement model given by Eq. (7.8).

few moments of the initial state $\rho_{0}$ may be known in practice, and it is prudent to assume that $\rho_{0}$ is in the constrained family $\mathbf{G}_{\gamma}$ defined by Eq. (5.1). The densityoperator family for the problem can be expressed as

$$
\mathbf{G}=\left\{\rho\left(\beta, \rho_{0}\right)=\mathcal{U}_{\beta} \rho_{0}: \beta \in \Theta_{\beta} \subseteq \mathbb{R}, \rho_{0} \in \mathbf{G}_{\gamma}\right\},
$$

where the unitary map $\mathcal{U}_{\beta}$ is defined as

$$
\mathcal{U}_{\beta} \rho_{0} \equiv \exp (-i H \beta) \rho_{0} \exp (i H \beta) \text {. }
$$

Generalization for more complicated generators is possible [12] but outside the scope of this paper.

Define an inner product and a norm with respect to the true $\rho_{0}$ as

$$
\left\langle h_{1}, h_{2}\right\rangle_{0} \equiv \operatorname{tr} \rho_{0} h_{1} \circ h_{2}, \quad\|h\|_{0} \equiv \sqrt{\langle h, h\rangle_{0}} .
$$

Define also the operator Hilbert space $\mathcal{Z}_{0}$ with respect to $\rho_{0}$, the tangent space $\mathcal{T}_{0}$ at $\rho_{0}$ with respect to $\mathbf{G}_{\gamma}$, and the orthocomplement $\mathcal{T}_{0}^{\perp}$ that gives $\mathcal{Z}_{0}=\mathcal{T}_{0} \oplus \mathcal{T} \perp$, in the same way as how the spaces $\mathcal{Z}, \mathcal{T}$, and $\mathcal{T}^{\perp}$ are defined with respect to $\rho$. Noting the unitarity of $\mathcal{U}_{\beta}$ and following the method in Appendix $\mathrm{C}$, it can be shown that the nuisance tangent space is given by

$$
\Lambda=\mathcal{U}_{\beta} \mathcal{T}_{0} \equiv\left\{\mathcal{U}_{\beta} h: h \in \mathcal{T}_{0}\right\} .
$$

Define the map adjoint to $\mathcal{U}_{\beta}$ by $\mathcal{U}_{\beta}^{*} h \equiv \exp (i H \beta) h$ $\exp (-i H \beta)$. Exploiting the isomorphism between $\Lambda$ and $\mathcal{T}_{0}$, we can compute the efficient score as follows:

$$
\begin{aligned}
S_{\text {eff }} & =S^{\beta}-\Pi\left(S^{\beta} \mid \Lambda\right)=S^{\beta}-\mathcal{U}_{\beta} \Pi\left(\mathcal{U}_{\beta}^{*} S^{\beta} \mid \mathcal{T}_{0}\right) \\
& =S^{\beta}-\mathcal{U}_{\beta}\left[\mathcal{U}_{\beta}^{*} S^{\beta}-\Pi\left(\mathcal{U}_{\beta}^{*} S^{\beta} \mid \mathcal{T} \perp\right)\right] \\
& =\mathcal{U}_{\beta} \Pi\left(\mathcal{U}_{\beta}^{*} S^{\beta} \mid \mathcal{T}_{0}^{\perp}\right) \\
& =\left\langle R, \mathcal{U}_{\beta}^{*} S^{\beta}\right\rangle_{0}^{\top}\langle R, R\rangle_{0}^{-1} \mathcal{U}_{\beta} R,
\end{aligned}
$$

where $R$ is the vector of antiscores with respect to $\rho_{0}$, as defined by Eq. (5.4) but with $\rho_{0}$ and $\langle\cdot, \cdot\rangle_{0}$ instead. Equation (7.15) can be further simplified, with

$$
\begin{aligned}
\left\langle R, \mathcal{U}_{\beta}^{*} S^{\beta}\right\rangle_{0} & =\left\langle\mathcal{U}_{\beta} R, S^{\beta}\right\rangle \\
& =-i \operatorname{tr} \rho\left[\mathcal{U}_{\beta} R, H\right] \\
& =-i \operatorname{tr} \rho_{0}[R, H]=[R, H]_{0},
\end{aligned}
$$

where $[A, B]_{j k} \equiv A_{j} B_{k}-B_{k} A_{j}$ and $[\cdot, \cdot]_{0}$ is shorthand for $-i \operatorname{tr} \rho_{0}[\cdot, \cdot]$. Equation (7.17) comes from the fact that $S^{\beta}=\mathfrak{D} H$ for the model given by Eq. (7.9), where $\mathfrak{D}$ is the so-called commutation superoperator defined by [7,25]

$$
\langle h, \mathfrak{D} H\rangle=-i \operatorname{tr} \rho[h, H] \forall h \in \mathcal{Y} .
$$

The final result is

$$
\begin{gathered}
S_{\text {eff }}=[R, H]_{0}^{\top}\langle R, R\rangle_{0}^{-1} \mathcal{U}_{\beta} R, \\
\left\|S_{\text {eff }}\right\|^{2}=[R, H]_{0}^{\top}\langle R, R\rangle_{0}^{-1}[R, H]_{0}, \\
\tilde{\mathrm{H}}^{(N)}=\frac{1}{N\left\|S_{\text {eff }}\right\|^{2}} .
\end{gathered}
$$

In particular, if the constraint is linear and a scalar given by

$$
\operatorname{tr} \rho_{0} Z=0, \quad R=Z,
$$

then

$$
\tilde{\mathrm{H}}^{(N)}=\frac{\|Z\|_{0}^{2}}{N[Z, H]_{0}^{2}},
$$

which gives Eq. (2.7). $\|Z\|_{0}^{2}$ is the variance of $Z$, while

$$
[Z, H]_{0}=-i \operatorname{tr} \rho_{0}[Z, H]=\left.\operatorname{tr} \rho_{0} \frac{\partial}{\partial \beta} \mathcal{U}_{\beta}^{*} Z\right|_{\beta=0}
$$

is a measure of how sensitive the Heisenberg-picture $Z$ is to the displacement. An intuitive explanation of this result is as follows. A displacement can be estimated only with respect to a known reference. If only the mean of $Z$ is known about the initial state, then it is the only reference in the quantum object that is available to the observer. It is therefore not surprising — in hindsight - that the statistics of $Z$ determine the fundamental limit.

If $H$ is the momentum operator and $Z$ is the position operator satisfying $[Z, H]=i$, the Heisenberg picture of $Z$ is

$$
\mathcal{U}_{\beta}^{*} Z=\beta+Z,
$$

which is a quantum additive-noise model with no known statistics about the noise operator $Z$ other than its mean. Measurements of $Z$ and the sample mean of the outcomes are efficient. This problem then becomes equivalent to the $\beta=\operatorname{tr} \rho Y$ example, but note that Eqs. (7.21) and (7.22) are 
more general, as they can deal with any generator, a $\beta$ that cannot be easily expressed as a functional of $\rho$, and more general constraints.

Another example is optical phase estimation with

$$
H=a^{\dagger} a=\frac{1}{2}\left(Z_{1}^{2}+Z_{2}^{2}\right)
$$

and constraint $\operatorname{tr} \rho_{0} Z=\zeta$ on the mean of the quadrature operators $Z=\left(Z_{1}, Z_{2}\right)^{\top}$ with $\left[Z_{1}, Z_{2}\right]=i$. There is no phase observable [52], so expressing $\beta$ as a functional of $\rho$ is difficult, if not impossible. Equations (7.21) and (7.22), on the other hand, are simple expressions in terms of the generator and the antiscores. In Eqs. (7.20)-(7.22), $R=Z-\zeta$,

$$
\left\langle R_{j}, R_{k}\right\rangle_{0}=\operatorname{tr} \rho_{0}\left(Z_{j}-\zeta_{j}\right) \circ\left(Z_{k}-\zeta_{k}\right)
$$

is simply the covariance matrix of the quadratures, while

$$
\begin{gathered}
{\left[R_{1}, H\right]_{0}=\left[Z_{1}, H\right]_{0}=\operatorname{tr} \rho_{0} Z_{2}=\zeta_{2},} \\
{\left[R_{2}, H\right]_{0}=\left[Z_{2}, H\right]_{0}=-\operatorname{tr} \rho_{0} Z_{1}=-\zeta_{1}}
\end{gathered}
$$

are the mean quadrature values. The efficient influence $\delta_{\text {eff }} \propto S_{\text {eff }}$ is a linear combination of the quadratures according to Eq. (7.20), indicating the ideal, though parameter-dependent, quadrature to be measured. An adaptive measurement can then aim to measure the ideal quadrature to approach the quantum limit.

When $\rho_{0}$ is exactly known, the Helstrom bound for displacement estimation has been computed exactly only if $\rho_{0}$ is pure or Gaussian. Only looser bounds have been found otherwise [1,2,7]. The Mandelstam-Tamm inequality, for example, is looser than the Helstrom bound for mixed states [7]. $S^{\beta}$ is determined by $\mathfrak{D} H$, and if $\rho_{0}$ is a high-dimensional non-Gaussian mixed state, $S^{\beta}$ is intractable. With the infinitely many nuisance parameters and infinitely many scores assumed here, the problem is hopeless under the conventional bottom-up approach. The top-down geometric approach, on the other hand, is able to avoid the computation of the scores altogether and give a simple result in terms of the more tractable antiscores.

\section{VECTORAL PARAMETER OF INTEREST}

To complete the formalism, here, we generalize the core results in this paper for a vectoral parameter of interest $\beta \in \mathbb{R}^{q}$ with $q \geq 1$ entries. $p$, the dimension of the parameter space, should be at least as large as $q$ and may be infinite. Define the error matrix as

$$
\Sigma \equiv \int[\check{\beta}(\lambda)-\beta][\check{\beta}(\lambda)-\beta]^{\top} \operatorname{tr} d E(\lambda) \rho,
$$

where $\check{\beta}: \mathcal{X} \rightarrow \mathbb{R}^{q}$ is an estimator. An influence operator should then be a vector of $q$ operators. The inner product between two vectoral operators and the norm are now defined as

$\operatorname{tr}\langle h, g\rangle=\sum_{j=1}^{q} \operatorname{tr} \rho\left(h_{j} \circ g_{j}\right), \quad\|h\| \equiv \sqrt{\operatorname{tr}\langle h, h\rangle}$.

The Hilbert spaces $\mathcal{Y}$ and $\mathcal{Z}$ for the vectoral operators are still expressed as Eqs. (3.13) and (3.14), while the tangent space is now defined as the replicating space [21]

$\mathcal{T} \equiv(\overline{\operatorname{span}}\{S\})^{\oplus q} \equiv \underbrace{\overline{\operatorname{span}}\{S\} \oplus \cdots \oplus \overline{\operatorname{span}}\{S\}}_{q \text { terms }}$.

The set of influence operators is still given by Eq. (3.21) if $\langle S, \delta\rangle=\partial \beta$ is interpreted as $\left\langle S^{\sigma}, \delta_{k}\right\rangle=\partial \beta_{k}$ for all submodels and $k=1, \ldots, q$. For an unbiased measurement, the error operator given by Eq. (3.22) remains an element of $\mathcal{D}$, and it can be shown (see Sec. 6.2 in Ref. [7]) that

$$
\Sigma \geq\langle\delta, \delta\rangle
$$

where the matrix inequality $A \geq B$ means that $A-B$ is positive semidefinite. The GHB can then be expressed as

$$
\mathrm{E} \equiv \operatorname{tr} W \Sigma \geq \operatorname{tr} W\langle\delta, \delta\rangle \geq \inf _{\delta \in \mathcal{D}} \operatorname{tr} W\langle\delta, \delta\rangle \equiv \tilde{\mathrm{H}}
$$

where $W \geq 0$ is a real cost matrix [8]. Generalizing Theorems 1 and 3, we have the following theorem.

Theorem 7.-The GHB for a vectoral parameter of interest is given by

$$
\tilde{\mathrm{H}}=\min _{\delta \in \mathcal{D}} \operatorname{tr} W\langle\delta, \delta\rangle=\operatorname{tr} W\left\langle\delta_{\text {eff }}, \delta_{\text {eff }}\right\rangle,
$$

where the efficient influence $\delta_{\text {eff }}$ is the unique element in $\mathcal{D}$ given by

$$
\delta_{\text {eff }}=\Pi(\delta \mid \mathcal{T}) .
$$

Proof.-The proof is delegated to Appendix J.

It is straightforward to generalize the methods introduced in this paper to compute the GHB for the vectoral case.

Holevo proposed another bound, denoted in the following by the sans-serif $X$, that can account for the quantum effect of observable incompatibility in multiparameter estimation $[7,40]$. Before we prove the bound and related results, we need the following lemma.

Lemma 1-(Belavkin and Grishanin [65]).-For any complex positive-semidefinite matrix $A$,

$$
\operatorname{tr} \operatorname{Re} A \geq\|\operatorname{Im} A\|_{1}
$$


where $\operatorname{Re} A$ and $\operatorname{Im} A$ denote the entrywise real and imaginary parts of $A$, respectively, and $\|\cdot\|_{1}$ denotes the trace norm, defined as the sum of the singular values.

Proof.-The proof is provided in Appendix $\mathrm{K}$ for completeness.

We can now present the Holevo bound. It requires little modification to be applied to semiparametric estimation; only the definition of $\mathcal{D}$ needs to be generalized to Eq. (3.21) here. Otherwise, the proof is standard $[7,28,40]$; we provide it here simply to demonstrate that it remains valid in the semiparametric setting.

Theorem 8.-

$\mathrm{E} \geq \mathrm{X} \equiv \inf _{\delta \in \mathcal{D}}\left[\operatorname{tr} W \operatorname{Re} \Gamma(\delta)+\|\sqrt{W} \operatorname{Im} \Gamma(\delta) \sqrt{W}\|_{1}\right]$,

where $\Gamma(\delta)$ is a complex matrix given by

$$
\Gamma_{j k}(\delta) \equiv \operatorname{tr} \rho \delta_{j} \delta_{k}
$$

Proof.-Holevo proved [see Eq. (6.6.55) in Ref. [7]] that the error matrix and the error operator of any unbiased measurement obey

$$
\Sigma \geq \Gamma(\delta) .
$$

Thus, $A=\sqrt{W}(\Sigma-\Gamma) \sqrt{W} \geq 0$. Applying Lemma 1 and noting that $\Sigma$ is real, we obtain

$$
\begin{aligned}
\operatorname{tr} \operatorname{Re} A & =\operatorname{tr} \sqrt{W}(\Sigma-\operatorname{Re} \Gamma) \sqrt{W}=\operatorname{tr} W(\Sigma-\operatorname{Re} \Gamma) \\
& \geq\|\operatorname{Im}[\sqrt{W}(\Sigma-\Gamma) \sqrt{W}]\|_{1}=\|\sqrt{W} \operatorname{Im} \Gamma \sqrt{W}\|_{1} .
\end{aligned}
$$

Hence,

$$
\operatorname{tr} W \Sigma \geq \operatorname{tr} W \operatorname{Re} \Gamma+\|\sqrt{W} \operatorname{Im} \Gamma \sqrt{W}\|_{1} \geq \mathrm{X} .
$$

The asymptotic attainability of the Holevo bound for $d<\infty$ is shown in Refs. [28-30]. The rough idea there is to consider a two-step method: First, find an estimate $\breve{\theta}$ of $\theta$ using some of the object copies, and then perform a measurement based on the influence operators obtained from the minimization in Eq. (8.9), assuming $\check{\theta}$ to be the truth. In the limit of $N \rightarrow \infty$, the overhead for finding $\check{\theta}$ is benign, and it can be shown that the error approaches $\mathrm{X}$ by local asymptotic normality.

For all the examples studied in previous sections, $\beta$ is a scalar, and it is straightforward to prove that the Holevo bound is equal to the GHB in that case.

Corollary 4.-If $\beta$ is a scalar $(q=1)$,

$$
X=\tilde{H} .
$$

Proof.-For $q=1, \Gamma(\delta)=\operatorname{tr} \rho \delta^{2}$ and $\operatorname{Im} \Gamma(\delta)=0$, leading to

$$
\mathrm{X}=\inf _{\delta \in \mathcal{D}} \operatorname{tr} W \operatorname{Re} \Gamma(\delta)=\inf _{\delta \in \mathcal{D}} \operatorname{tr} W\langle\delta, \delta\rangle=\tilde{\mathrm{H}} .
$$

The scalar GHB hence inherits all the properties of the Holevo bound, including its asymptotic attainability. In fact, for any $q$, the Holevo bound turns out to be a marginal improvement over the GHB only.

Theorem 9.-

$$
\tilde{\mathrm{H}} \leq \mathrm{X} \leq 2 \tilde{\mathrm{H}} .
$$

Proof.-For all $\delta \in \mathcal{D}$,

$$
\begin{aligned}
& \operatorname{tr} W \operatorname{Re} \Gamma(\delta)+\|\sqrt{W} \operatorname{Im} \Gamma(\delta) \sqrt{W}\|_{1} \\
& \geq \operatorname{tr} W \operatorname{Re} \Gamma(\delta)=\operatorname{tr} W\langle\delta, \delta\rangle \geq \tilde{\mathrm{H}}
\end{aligned}
$$

As $X$ is the infimum of Eq. (8.17), we obtain $X \geq \tilde{H}$, the first inequality of the theorem. The second inequality is proved as follows:

$$
\begin{aligned}
X & \leq \operatorname{tr} W \operatorname{Re} \Gamma\left(\delta_{\text {eff }}\right)+\left\|\sqrt{W} \operatorname{Im} \Gamma\left(\delta_{\text {eff }}\right) \sqrt{W}\right\|_{1} \equiv \mathrm{D} \\
& \leq \operatorname{tr} W \operatorname{Re} \Gamma\left(\delta_{\text {eff }}\right)+\operatorname{tr} \sqrt{W} \operatorname{Re} \Gamma\left(\delta_{\text {eff }}\right) \sqrt{W} \\
& =2 \operatorname{tr} W \operatorname{Re} \Gamma\left(\delta_{\text {eff }}\right)=2 \tilde{\mathrm{H}},
\end{aligned}
$$

where Eq. (8.20) is obtained by applying Lemma 1 to $A=\sqrt{W} \Gamma\left(\delta_{\text {eff }}\right) \sqrt{W}$.

The first inequality $\tilde{H} \leq X$ is well known $[7,39,40]$. A special case $\mathrm{X} \leq 2 \mathrm{H}$ of the second inequality-when $p<\infty, K^{-1}$ exists, and $\tilde{\mathrm{H}}=\mathrm{H}$ is the original Helstrom bound-was proved recently in Ref. [35]. $\mathrm{X}=2 \mathrm{H}$ can be attained in special cases [28-30].

Theorem 9 implies that the effect of incompatibility is surprisingly benign in the context of asymptotic statistics, the GHB can be approached to within a factor of 2 if the Holevo bound is attainable, and the GHB is a serviceable alternative to the Holevo bound, especially when the latter is more difficult to compute. See Ref. [28] for further interesting discussions regarding this result.

As an aside, we remark that the $D$ in Eq. (8.19) is called the $\mathfrak{D}$-invariant bound and coincides with $\mathrm{X}$ if $\mathcal{T}=\mathfrak{D} \mathcal{T}$, where $\mathfrak{D}$ is given by Eq. (7.19) $[7,66]$. In general, $D$ offers a tighter upper bound on $\mathrm{X}$ than $2 \tilde{\mathrm{H}}$ but may not be much more difficult to compute, as it also depends on $\delta_{\text {eff }}$, which can be found via the methods introduced in this work.

We present a few other interesting results concerning multiparameter estimation with $p<\infty$ in Appendix L. 
Finally, we generalize the concept of efficient score in Theorem 6 for a vectoral $\beta$.

Theorem 10.-Assume a density-operator family given by

$$
\mathbf{G}=\left\{\rho(\beta, \eta): \beta \in \Theta_{\beta} \subseteq \mathbb{R}^{q}, \eta \in \mathcal{G}\right\} .
$$

Let $S^{\beta}=\left(S_{1}^{\beta}, \ldots, S_{q}^{\beta}\right)^{\top}$ be the scores with respect to $\beta$ and $\left\{S^{\eta}\right\}$ be the nuisance tangent set. Assume the unbiasedness condition for influence operators $\delta \in \mathcal{D}$ given by

$$
\left\langle S^{\beta}, \delta\right\rangle=I, \quad\left\langle S^{\eta}, \delta\right\rangle=0,
$$

where $I$ is the identity matrix. The efficient influence and the GHB are given by

$$
\delta_{\text {eff }}=\left\langle S_{\text {eff }}, S_{\text {eff }}\right\rangle^{-1} S_{\text {eff }}, \quad \tilde{\mathrm{H}}=\operatorname{tr} W\left\langle S_{\text {eff }}, S_{\text {eff }}\right\rangle^{-1},
$$

respectively, where the efficient score $S_{\text {eff }}$ is given by

$$
S_{\text {eff }}=S^{\beta}-\Pi\left(S^{\beta} \mid \Lambda\right), \quad \Lambda \equiv\left(\overline{\operatorname{span}}\left\{S^{\eta}\right\}\right)^{\oplus q},
$$

and $\left\langle S_{\text {eff }}, S_{\text {eff }}\right\rangle^{-1}$ is assumed to exist.

Proof.-The proof is almost identical to that of Theorem 6 in Appendix I and omitted here for brevity.

\section{CONCLUSION}

We have founded a theory of quantum semiparametric estimation and showcased its power by producing simple quantum bounds for a large class of problems with high dimensions and few assumptions about the density operator. The theory establishes the notion of quantum semiparametric efficiency, which should inform and inspire the design of more efficient measurements in many areas of quantum physics.

While the experimental design of efficient semiparametric measurements is only touched upon here and awaits further research, the importance of the quantum limits set forth should not be underestimated. As more experiments are now being performed on complex quantum systems and advantages of such systems for metrology and information processing, in general, are being claimed, the precision limits serve as ultimate yardsticks as well as "no-go" theorems that guard against spurious proposals and fruitless endeavors, in the same way the laws of thermodynamics impose limits to engines and rule out perpetual-motion machines. Deriving precision limits for highly complex or poorly modeled quantum systems was a daunting task under the curse of dimensionality; the semiparametric theory offers a new way forward.

Many open problems still remain. More extensions and applications of the theory remain to be worked out. The asymptotic attainability of efficiency $[8,9,28-30]$ is a thorny issue for infinite-dimensional problems. The assumption of unbiased estimation is a drawback; generalizations to the
Bayesian or minimax paradigm [67] should help but await further research. These problems should benefit from studies of alternative quantum bounds beyond the Cramér-Rao type [68]. In view of Eq. (3.52) and Figs. 3 and 4, the connections of quantum semiparametrics to other domains of quantum information [43] and quantum state geometry $[8,9,26]$ are also interesting future directions.

In light of the richness and wide applications of the classical semiparametric theory [19-24], this work has only scratched the surface of the full potential of quantum semiparametrics. It should open doors to further useful results.

\section{ACKNOWLEDGMENTS}

We thank M. G. Genoni both for several fruitful discussions and for making us aware of Ref. [35]. We are grateful to R. Nair, M. Guţă, R. Gill, D. Branford, R. Demkowicz-Dobrańzski, J.F. Friel, W. Górecki, and J. Suzuki for useful discussions. This research is partly supported by the National Research Foundation (NRF) Singapore, under its Quantum Engineering Programme (Grant No. QEP-P7). A. D. and F. A. have been supported by the United Kingdom Engineering and Physical Sciences Research Council (EP/K04057X/2) and the United Kingdom National Quantum Technologies Programme (EP/M01326X/1 and EP/M013243/1). F. A. also acknowledges financial support from the National Science Center (Poland) Grant No. 2016/22/E/ST2/00559.

\section{APPENDIX A: PROOF OF COROLLARY 1}

If $p<\infty$ and $K^{-1}$ exists, the solution to $\Pi(\delta \mid \mathcal{T})$ can be found, for example, in Ref. [20] [Eq. (15) in Appendix A.2]. Here, we give a simple proof for completeness. By definition of the projection [37],

$$
\Pi(\delta \mid \mathcal{T})=\underset{h \in \mathcal{T}}{\arg \min }\|\delta-h\| .
$$

Any $h \in \mathcal{T}$ can be expressed as the linear combination $w^{\top} S$ with respect to a certain vector $w \in \mathbb{R}^{p}$. Then

$$
\|\delta-h\|^{2}=\langle\delta, \delta\rangle-w^{\top}\langle S, \delta\rangle-\langle S, \delta\rangle^{\top} w+w^{\top}\langle S, S\rangle w .
$$

The solution to the least-squares problem is

$$
\begin{gathered}
w_{\min }=\langle S, S\rangle^{-1}\langle S, \delta\rangle, \\
\Pi(\delta \mid \mathcal{T})=w_{\min }^{\top} S=\langle S, \delta\rangle^{\top}\langle S, S\rangle^{-1} S .
\end{gathered}
$$

Hence,

$$
\|\Pi(\delta \mid \mathcal{T})\|^{2}=\langle S, \delta\rangle^{\top}\langle S, S\rangle^{-1}\langle S, \delta\rangle,
$$


which is equal to Eq. (3.8), since $\langle S, \delta\rangle=\partial \beta$ for an influence operator.

\section{APPENDIX B: PROOF OF COROLLARY 2}

Denote any concept discussed so far with the superscript $(N)$ if it is associated with $\mathbf{F}^{(N)}$, but omit the superscript (1) for brevity if $N=1$. From $\mathcal{Z}$, we generate a subspace $U \mathcal{Z} \subset \mathcal{Z}^{(N)}$ such that

$$
U \mathcal{Z} \equiv\{U h: h \in \mathcal{Z}\}
$$

$U$ is a surjective map to $U \mathcal{Z}$ by definition of the space. It can be shown that

$$
\left\langle U h_{1}, U h_{2}\right\rangle^{(N)}=\left\langle h_{1}, h_{2}\right\rangle \forall h_{1}, h_{2} \in \mathcal{Z},
$$

so $U \mathcal{Z}$ is isomorphic to $\mathcal{Z}$, and $U$ is a unitary map from $\mathcal{Z}$ to $U \mathcal{Z}$ [38]. It can also be shown that

$$
S^{(N)}=\sqrt{N} U S,
$$

so $\mathcal{T}^{(N)}=\overline{\operatorname{span}}\left\{S^{(N)}\right\} \subseteq U \mathcal{Z}$, and $\mathcal{T}^{(N)}$ is isomorphic to $\mathcal{T}$. For any $U h \in U \mathcal{Z}$, it is not difficult to prove that

$$
\Pi\left(U h \mid \mathcal{T}^{(N)}\right)=U \Pi(h \mid \mathcal{T}),
$$

given the isomorphisms. Now, let

$$
\delta^{(N)}=\frac{U \delta}{\sqrt{N}} \in U \mathcal{Z},
$$

where $\delta$ is an influence operator. $\delta^{(N)}$ is also an influence operator, since

$\left\langle S^{(N)}, \delta^{(N)}\right\rangle^{(N)}=\left\langle\sqrt{N} U S, \frac{U \delta}{\sqrt{N}}\right\rangle^{(N)}=\langle S, \delta\rangle=\partial \beta$.

The efficient influence for $\mathbf{F}^{(N)}$ becomes

$\delta_{\text {eff }}^{(N)}=\Pi\left(\delta^{(N)} \mid \mathcal{T}^{(N)}\right)=\frac{\Pi\left(U \delta \mid \mathcal{T}^{(N)}\right)}{\sqrt{N}}=\frac{U \Pi(\delta \mid \mathcal{T})}{\sqrt{N}}=\frac{U \delta_{\text {eff }}}{\sqrt{N}}$,

the norm becomes

$$
\left\|\delta_{\mathrm{eff}}^{(N)}\right\|^{(N)}=\frac{\left\|\delta_{\mathrm{eff}}\right\|}{\sqrt{N}}
$$

and the corollary ensues.

\section{APPENDIX C: PROOF OF COROLLARY 3}

Let $\left\{S^{(N)}\right\}$ be the tangent set for $\mathbf{G}^{(N)}$. For each parametric submodel $\{\sigma(\theta)\}$ of $\mathbf{G}$, let

$$
\left\{\tau(\theta)=\sigma(\theta)^{\otimes N}\right\}
$$

be a parametric submodel of $\mathbf{G}^{(N)}$. The score of the submodel is given by

$$
S^{\tau}=\sqrt{N} U S^{\sigma} .
$$

In other words, each $S^{\sigma} \in\{S\}$ can be used to generate a score in $\left\{S^{(N)}\right\}$ via Eq. (C2). The set of scores generated this way is therefore a subset of $\left\{S^{(N)}\right\}$, viz.,

$$
\{\sqrt{N} U S\} \equiv\left\{\sqrt{N} U S^{\sigma}: S^{\sigma} \in\{S\}\right\} \subseteq\left\{S^{(N)}\right\} .
$$

Conversely, any parametric submodel of $\mathbf{G}^{(N)}$ must be in the form of Eq. (C1), with $\{\sigma(\theta)\}$ being a certain parametric submodel of $\mathbf{G}$. The score of the former is then related to the score of the latter via Eq. (C2). Since $\{S\}$ includes the scores of all parametric submodels of $\mathbf{G}$, any $S^{\tau} \in\left\{S^{(N)}\right\}$ must be in $\{\sqrt{N} U S\}$. Thus, $\left\{S^{(N)}\right\} \subseteq$ $\{\sqrt{N} U S\}$, and equality holds, viz.,

$$
\left\{S^{(N)}\right\}=\{\sqrt{N} U S\} .
$$

It follows that

$$
\mathcal{T}^{(N)} \equiv \overline{\operatorname{span}}\left\{S^{(N)}\right\}=\overline{\operatorname{span}}\{\sqrt{N} U S\}
$$

is isomorphic to $\mathcal{T}=\overline{\operatorname{span}}\{S\}$. Hence, projecting an influence operator of the form $\delta^{(N)}=U \delta / \sqrt{N}$ into $\mathcal{T}^{(N)}$ gives the efficient influence $\delta_{\text {eff }}^{(N)}=U \delta_{\text {eff }} / \sqrt{N}$, by the same argument as Appendix B.

\section{APPENDIX D: THE SET OF BOUNDED OPERATORS IS DENSE IN $\mathcal{Z}$}

To generalize Theorem 2 for the infinite-dimensional case and prove Theorem 4, we need to be mindful of the unbounded operators in $\mathcal{Z}$. The good news is that they are well defined as limits of bounded-operator sequences in $\mathcal{Y}$, thanks to Holevo [7,25]; just a minor modification is needed to make his result work for $\mathcal{Z}$.

Consider the set $\mathcal{B}$ of bounded elements defined by Eq. (4.13). If $d<\infty, \mathcal{B}=\overline{\mathcal{B}}=\mathcal{Z}$, since all operators are bounded in the finite-dimensional case, but if $d=\infty$, $\mathcal{B} \subset \mathcal{Z}$ is a strict subset. A useful lemma is as follows.

Lemma $2 .-\overline{\mathcal{B}}=\mathcal{Z}$.

Proof.-Theorem 2.8.1 in Ref. [7] implies that, for any $h \in \mathcal{Z} \subset \mathcal{Y}$, there exists a Cauchy sequence $\left\{h_{n}\right\}$ with each $h_{n} \in \mathcal{Y}$ satisfying $\left\|h_{n}\right\|_{\text {op }}<\infty$ such that

$$
\lim _{n \rightarrow \infty}\left\|h-h_{n}\right\|=0 .
$$

To derive a similar convergent sequence in $\mathcal{Z}$, consider the projection of each $h_{n}$ into $\mathcal{Z}$, written as 


$$
h_{n}^{\prime}=\Pi\left(h_{n} \mid \mathcal{Z}\right)=h_{n}-\left\langle h_{n}, I\right\rangle \in \mathcal{Z} .
$$

Denote a bounded operator in the equivalence class of $h_{n}$ as $\hat{h}_{n}$. An operator for $h_{n}^{\prime}$ can be expressed as

$$
\hat{h}_{n}^{\prime}=\hat{h}_{n}-\left\langle h_{n}, I\right\rangle \hat{I} \text {. }
$$

Since $\left\|\hat{h}_{n}\right\|_{\text {op }}<\infty$ and $\left\|\left\langle h_{n}, I\right\rangle \hat{I}\right\|_{\text {op }}=\left|\left\langle h_{n}, I\right\rangle\right|<\infty$,

$$
\left\|\hat{h}_{n}^{\prime}\right\|_{\text {op }} \leq\left\|\hat{h}_{n}\right\|_{\text {op }}+\left\|\left\langle h_{n}, I\right\rangle \hat{I}\right\|_{\text {op }}<\infty
$$

by the triangle inequality, leading to $h_{n}^{\prime} \in \mathcal{B}$. The Pythagorean theorem leads to

$$
\begin{gathered}
\left\|h_{n}-h_{m}\right\| \geq\left\|h_{n}^{\prime}-h_{m}^{\prime}\right\| \forall n, m, \\
\left\|h-h_{n}\right\| \geq\left\|h-h_{n}^{\prime}\right\|,
\end{gathered}
$$

which can be combined with Eq. (D1) to give

$$
\lim _{n \rightarrow \infty}\left\|h-h_{n}^{\prime}\right\|=0 .
$$

In other words, $\left\{h_{n}^{\prime}\right\}$, with each $h_{n}^{\prime} \in \mathcal{B}$, is also Cauchy and converges to $h$. As the argument applies to any $h \in \mathcal{Z}, \mathcal{B}$ is dense in $\mathcal{Z}$, and the closure of $\mathcal{B}$ gives $\mathcal{Z}$.

\section{APPENDIX E: PROOF OF PROPOSITION 1}

Let the orthocomplement of $\mathcal{V}$ in $\mathcal{T}$ be $\mathcal{V}_{\mathcal{T}}^{\perp}$. Then the Pythagorean theorem yields

$$
\begin{aligned}
\left\|\delta_{\text {eff }}\right\|^{2} & =\left\|\Pi\left(\delta_{\text {eff }} \mid \mathcal{V}\right)\right\|^{2}+\left\|\Pi\left(\delta_{\text {eff }} \mid \mathcal{V}_{\mathcal{T}}^{\perp}\right)\right\|^{2} \\
& \geq\left\|\Pi\left(\delta_{\text {eff }} \mid \mathcal{V}\right)\right\|^{2}=\|\Pi(\Pi(\delta \mid \mathcal{T}) \mid \mathcal{V})\|^{2} \\
& =\|\Pi(\delta \mid \mathcal{V})\|^{2},
\end{aligned}
$$

where the last step uses Proposition 3B in Appendix A.2 in Ref. [20]. $\|\Pi(\delta \mid \mathcal{V})\|^{2}=\|\delta\|^{2}-\left\|\Pi\left(\delta \mid \mathcal{V}^{\perp}\right)\right\|^{2}$ follows again from the Pythagorean theorem for a $\delta \in \mathcal{Z}=\mathcal{V} \oplus \mathcal{V}^{\perp}$. Equation (5.23) comes from Theorem 1.

\section{APPENDIX F: PROOF OF PROPOSITION 2}

Let $P$ be the true density. For real functions on $\mathbb{C}$, define an inner product and a norm with respect to $P$ as, respectively,

$$
\langle f, g\rangle_{P} \equiv \int d^{2} \alpha P(\alpha) f(\alpha) g(\alpha), \quad\|f\|_{P} \equiv \sqrt{\langle f, f\rangle_{P}}
$$

Define the Hilbert space of zero-mean functions as

$$
\mathcal{Z}_{P} \equiv\left\{f:\|f\|_{P}<\infty,\langle f, 1\rangle_{P}=0\right\} .
$$

For each $f \in \mathcal{Z}_{P}$, construct the parametric submodel

$$
\sigma(\theta)=\int d^{2} \alpha P(\alpha \mid \theta)|\alpha\rangle\langle\alpha|
$$

$$
P(\alpha \mid \theta)=\frac{\{1+\tanh [f(\alpha) \theta]\} P(\alpha)}{\int d^{2} \alpha\{1+\tanh [f(\alpha) \theta]\} P(\alpha)},
$$

with the truth at $\sigma(0)=\rho$ and $P(\alpha \mid 0)=P(\alpha) . f(\alpha)$ is the score function with respect to $P(\alpha \mid \theta)$. The score with respect to $\sigma$ is then given by

$$
\rho \circ S=\rho \circ(\mathcal{E} f)=\int d^{2} \alpha P(\alpha) f(\alpha)|\alpha\rangle\langle\alpha|,
$$

where the map $\mathcal{E}: \mathcal{Z}_{P} \rightarrow \mathcal{Z}$ is a quantum version of the conditional expectation [8]. Hence,

$$
\left\{\mathcal{E} f: f \in \mathcal{Z}_{P}\right\} \subseteq\{S\} .
$$

Consider the inner product between $\mathcal{E} f$ and an $h \in \mathcal{B} \subset \mathcal{Z}$ given by

$$
\langle\mathcal{E} f, h\rangle=\operatorname{tr} \rho[(\mathcal{E} f) \circ h]=\operatorname{tr}[\rho \circ(\mathcal{E} f)] h=\left\langle f, \mathcal{E}^{*} h\right\rangle_{P},
$$

where Eq. (3.18) is used and $\mathcal{E}^{*}$ is the adjoint map given by the Husimi representation

$$
\left(\mathcal{E}^{*} h\right)(\alpha)=\langle\alpha|h| \alpha\rangle .
$$

Since $h \in \mathcal{Z}$,

$$
\operatorname{tr} \rho h=\int d^{2} \alpha P(\alpha)\langle\alpha|h| \alpha\rangle=\left\langle\mathcal{E}^{*} h, 1\right\rangle_{P}=0,
$$

and $\mathcal{E}^{*} h \in \mathcal{Z}_{P}$. The map $\mathcal{E}^{*}: \mathcal{B} \rightarrow \mathcal{Z}_{P}$ is obviously linear. It is also bounded, because

$$
\begin{aligned}
\left\|\mathcal{E}^{*} h\right\|_{P}^{2} & =\int d^{2} \alpha P(\alpha)(\langle\alpha|h| \alpha\rangle)^{2} \\
& \leq \int d^{2} \alpha P(\alpha)\left\langle\alpha\left|h^{2}\right| \alpha\right\rangle=\|h\|^{2} .
\end{aligned}
$$

Thus, $\mathcal{E}^{*}$ is a continuous linear map (see Theorem 1.5.7 in Ref. [37]). As $\mathcal{B}$ is a dense subset of $\mathcal{Z}$ by virtue of Lemma 2, $\mathcal{E}^{*}$ can be uniquely extended to a continuous linear map on the whole $\mathcal{Z}$ (see Theorem 1.5.10 in Ref. [37]).

Any $h \in \mathcal{T}^{\perp}$ must obey

$$
\langle\mathcal{E} f, h\rangle=\left\langle f, \mathcal{E}^{*} h\right\rangle_{P}=0 \forall f \in \mathcal{Z}_{P} .
$$

The only solution is $\mathcal{E}^{*} h=0$. In other words, $\mathcal{T}^{\perp}$ is in the null space of $\mathcal{E}^{*}$. As the Husimi representation is injective [69], the only solution to $\mathcal{E}^{*} h=0$ is $h=0$. Hence, $\mathcal{T}^{\perp}=\{0\}$, and $\mathcal{T}=\mathcal{Z}$. 


\section{APPENDIX G: $\mathcal{T}^{\perp}$ FOR DIFFRACTION-LIMITED INCOHERENT IMAGING IS INFINITE DIMENSIONAL}

Following Appendix F, it can be shown that $h \in \mathcal{T}^{\perp}$ if

$$
\left\langle\psi_{X}|h| \psi_{X}\right\rangle=0 \forall X \in \operatorname{supp} F
$$

for the incoherent-imaging problem in Sec. VI C. Consider, for example, $h=\int d k \tilde{h}(k)|k\rangle\langle k|$, where $|k\rangle$ is a momentum eigenket. Then, Eq. (G1) is satisfied if

$$
\left\langle\psi_{X}|h| \psi_{X}\right\rangle \propto \int d k \tilde{h}(k) \exp \left(-2 k^{2}\right)=0 .
$$

Let $\left\{\tilde{a}_{j}(k): j \in \mathbb{N}_{0}\right\}$ be the set of Hermite polynomials that are orthogonal with respect to the weight function $\exp \left(-2 k^{2}\right)$. Then, any $\tilde{a}_{j}(k)$ with $j>0$ satisfies Eq. (G2). Define the set

$$
\{a\} \equiv\left\{a_{j}=\int d k \tilde{a}_{j}(k)|k\rangle\langle k|: j \in \mathbb{N}_{1}\right\}
$$

Each $a_{j}$ obeys Eq. (G2) and

$$
\left\langle a_{j}, a_{k}\right\rangle \propto \int d k \tilde{a}_{j}(k) \tilde{a}_{k}(k) \exp \left(-2 k^{2}\right) \propto \delta_{j k},
$$

so $\{a\}$ is an orthogonal set with respect to the inner product given by Eq. (3.10). As $\overline{\operatorname{span}}\{a\} \subseteq \mathcal{T}^{\perp}$,

$$
\operatorname{dim} \mathcal{T}^{\perp} \geq|\{a\}|=\left|\mathbb{N}_{1}\right|,
$$

which means that the dimension of $\mathcal{T}^{\perp}$ must be infinite.

\section{APPENDIX H: DERIVATION OF EQ. (6.32)}

For the density-operator family given by Eq. (6.31), the extended convexity of the Helstrom information $[13,64]$ implies that

$$
\begin{gathered}
K \leq \tilde{K}=K^{g}+K^{h}, \\
K_{j k}^{g}=\left.\int d X F(X) \frac{\partial g\left(X \mid \theta_{g}\right)}{\partial \theta_{j}} \frac{\partial g\left(X \mid \theta_{g}\right)}{\partial \theta_{k}}\right|_{\theta=0}, \\
K_{j k}^{h}=\left.4\left\langle\Delta k^{2}\right\rangle \int d X F(X) \frac{\partial h\left(X \mid \theta_{h}\right)}{\partial \theta_{j}} \frac{\partial h\left(X \mid \theta_{h}\right)}{\partial \theta_{k}}\right|_{\theta=0},
\end{gathered}
$$

where $\left\langle\Delta k^{2}\right\rangle=\left\langle\psi_{0}\left|k^{2}\right| \psi_{0}\right\rangle-\left(\left\langle\psi_{0}|k| \psi_{0}\right\rangle\right)^{2}=1 / 4$ is the variance of $k$. With the explicit partition of $\theta$ into $\theta_{g}$ and $\theta_{h}, \tilde{K}$ can be expressed as

$$
\begin{aligned}
\tilde{K} & =\left(\begin{array}{cc}
K^{g} & 0 \\
0 & K^{h}
\end{array}\right) \\
& =\int d X F(X)\left(\begin{array}{cc}
\left(\partial_{g} g\right)\left(\partial_{g} g\right)^{\top} & 0 \\
0 & \left(\partial_{h} h\right)\left(\partial_{h} h\right)^{\top}
\end{array}\right),
\end{aligned}
$$

where $\partial_{g}=\left(\partial_{g 1}, \partial_{g 2}, \ldots\right)^{\top}$ and $\partial_{h}=\left(\partial_{h 0}, \partial_{h 1}, \ldots\right)^{\top}$. Let

$$
\begin{gathered}
g\left(X \mid \theta_{g}\right)=\sum_{j=1}^{\infty} \theta_{g j} a_{j}(X), \\
h\left(X \mid \theta_{h}\right)=X+\sum_{j=0}^{\infty} \theta_{h j} a_{j}(X),
\end{gathered}
$$

where $\left\{a_{j}(X): j \in \mathbb{N}_{0}\right\}$ is a set of orthogonal polynomials with respect to the true $F$ that satisfy $\int d X F(X) a_{j}(X) a_{k}(X)=\delta_{j k} \cdot a_{0}(X)=1$ is omitted from $g\left(X \mid \theta_{g}\right)$, because $g$ is a score function with respect to $F$ and $\int d X F(X) g\left(X \mid \theta_{g}\right)=0$ implies that $g\left(X \mid \theta_{g}\right)$ cannot contain $a_{0}(X)$ in its expansion. The orthonormality of $\{a\}$ leads to

$$
\tilde{K}=I, \quad \tilde{K}^{-1}=I .
$$

Now, consider

$$
\begin{gathered}
\beta_{\mu}(\theta)=\int d X F(X \mid \theta) X^{\mu} \\
=\int d Y G\left(Y \mid \theta_{g}\right)\left[h\left(Y \mid \theta_{h}\right)\right]^{\mu}, \\
\left.\frac{\partial \beta_{\mu}}{\partial \theta_{g j}}\right|_{\theta=0}=\int d X F(X) a_{j}(X) X^{\mu}, \\
\left.\frac{\partial \beta_{\mu}}{\partial \theta_{h j}}\right|_{\theta=0}=\mu \int d X F(X) X^{\mu-1} a_{j}(X) .
\end{gathered}
$$

Then,

$$
\begin{aligned}
\left(\partial \beta_{\mu}\right)^{\top} \tilde{K}^{-1} \partial \beta_{\mu} & =\sum_{j=1}^{\infty}\left(\frac{\partial \beta_{\mu}}{\partial \theta_{g j}}\right)^{2}+\sum_{j=0}^{\infty}\left(\frac{\partial \beta_{\mu}}{\partial \theta_{h j}}\right)^{2} \\
& =\beta_{2 \mu}-\beta_{\mu}^{2}+\mu^{2} \beta_{2 \mu-2}
\end{aligned}
$$

where the completeness property

$$
\sum_{j=0}^{\infty} a_{j}(X) a_{j}\left(X^{\prime}\right)=1+\sum_{j=1}^{\infty} a_{j}(X) a_{j}\left(X^{\prime}\right)=\delta\left(X-X^{\prime}\right)
$$

is assumed. With 


$$
\mathrm{H}_{\mu}^{\sigma}=\left(\partial \beta_{\mu}\right)^{\top} K^{-1} \partial \beta_{\mu} \geq\left(\partial \beta_{\mu}\right)^{\top} \tilde{K}^{-1} \partial \beta_{\mu},
$$

and using Corollary 3 and Proposition 1, Eq. (6.32) is obtained.

\section{APPENDIX I: PROOF OF THEOREM 6}

The proof follows the classical case [21]. As $S^{\beta} \notin \Lambda$, the $S_{\text {eff }}$ given by Eq. (7.7) is not zero. Let

$$
\delta=\frac{S_{\text {eff }}}{\left\|S_{\text {eff }}\right\|^{2}} .
$$

Notice that Eq. (7.7) is a projection of $S^{\beta}$ into a space orthogonal to $\Lambda$, so $S_{\text {eff }} \perp \Lambda$ and $\delta \perp \Lambda$. Then,

$$
\begin{gathered}
\left\langle S^{\beta}, \delta\right\rangle=\frac{1}{\left\|S_{\text {eff }}\right\|^{2}}\left\langle S_{\text {eff }}+\Pi\left(S^{\beta} \mid \Lambda\right), S_{\text {eff }}\right\rangle=1, \\
\left\langle S_{j}^{\eta}, \delta\right\rangle=0,
\end{gathered}
$$

because $\Pi\left(S^{\beta} \mid \Lambda\right) \in \Lambda$ and each $S_{j}^{\eta} \in \Lambda$. Thus, $\delta$ satisfies Eqs. (7.5) and is an influence operator. Notice also that $S_{\text {eff }}$ and $\delta$ are in $\mathcal{T}$, because $S^{\beta} \in \mathcal{T}$ and $\Pi\left(S^{\beta} \mid \Lambda\right) \in \Lambda \subseteq \mathcal{T}$. Hence, by Theorem 3,

$$
\delta_{\text {eff }}=\Pi(\delta \mid \mathcal{T})=\delta,
$$

and Eq. (I1) is the efficient influence.

\section{APPENDIX J: PROOF OF THEOREM 7}

We again follow Ref. [21]. Decompose any $\delta \in \mathcal{D} \subseteq$ $\mathcal{Z}=\mathcal{T} \oplus \mathcal{T}^{\perp}$ into

$$
\delta=\delta_{\text {eff }}+h, \quad \delta_{\text {eff }}=\Pi(\delta \mid \mathcal{T}), \quad h=\Pi\left(\delta \mid \mathcal{T}^{\perp}\right) .
$$

It is straightforward to prove that $\delta_{\text {eff }} \in \mathcal{D}$. As $h$ is orthogonal to any element in $\mathcal{T} \equiv(\overline{\operatorname{span}}\{S\})^{\oplus q}$, it must be orthogonal to $g=(0, \ldots, 0, e, 0, \ldots, 0)^{\top}$ with any $e \in$ $\overline{\operatorname{span}}\{S\}$ in any entry of $g$, say, the $j$ th entry. Then,

$$
\operatorname{tr}\langle h, g\rangle=\left\langle h_{j}, e\right\rangle=0,
$$

meaning that each entry of $h$ is orthogonal to $\overline{\operatorname{span}}\{S\}$. This condition leads to a stronger matrix form of the orthogonality between $\delta_{\text {eff }} \in \mathcal{T}$ and $h \in \mathcal{T}^{\perp}$ given by

$$
\left\langle\delta_{\mathrm{eff}}, h\right\rangle=0
$$

and a matrix form of the Pythagorean theorem given by

$$
\langle\delta, \delta\rangle=\left\langle\delta_{\text {eff }}, \delta_{\text {eff }}\right\rangle+\langle h, h\rangle \geq\left\langle\delta_{\text {eff }}, \delta_{\text {eff }}\right\rangle,
$$

resulting in Eq. (8.6). To prove the uniqueness of $\delta_{\text {eff }}$ in $\mathcal{D}$, suppose that there exists another $\delta^{\prime} \in \mathcal{D}$ that gives $\left\langle\delta^{\prime}, \delta^{\prime}\right\rangle=\left\langle\delta_{\text {eff }}, \delta_{\text {eff }}\right\rangle$. Define $g=\delta^{\prime}-\delta_{\text {eff }}$. As $\langle S, g\rangle=$ $\left\langle S, \delta^{\prime}\right\rangle-\left\langle S, \delta_{\text {eff }}\right\rangle=\partial \beta-\partial \beta=0, g \in \mathcal{T}^{\perp}$, and the matrix Pythagorean theorem gives $\left\langle\delta^{\prime}, \delta^{\prime}\right\rangle=\left\langle\delta_{\text {eff }}, \delta_{\text {eff }}\right\rangle+\langle g, g\rangle$. This fact implies that $\langle g, g\rangle=0,\|g\|^{2}=\operatorname{tr}\langle g, g\rangle=0$, and $g=0$, contradicting the assumption that $\delta^{\prime} \neq \delta_{\text {eff }}$. Hence, $\delta_{\text {eff }}$ must be unique.

\section{APPENDIX K: PROOF OF LEMMA 1}

Let the superscript $*$ denote the entrywise conjugation of a matrix and the superscript $\dagger=* \top$ denote the conjugate transpose. $A \geq 0$ means that $z^{\dagger} A z \geq 0$ for any $z \in \mathbb{C}^{q}$. We also have $A^{*} \geq 0$, since $z^{\dagger} A^{*} z=\left(z^{* \dagger} A z^{*}\right)^{*}=z^{* \dagger} A z^{*} \geq 0$ for any $z \in \mathbb{C}^{q}$. Thus, for any $z \in \mathbb{C}^{q}$,

$z^{\dagger}(\operatorname{Re} A \pm i \operatorname{Im} A) z \geq 0, \quad z^{\dagger}(\operatorname{Re} A) z \geq\left|z^{\dagger}(i \operatorname{Im} A) z\right|$.

Let $\left\{\lambda_{s}, z_{s}: s=1, \ldots, q\right\}$ be the eigenvalues and eigenvectors of the Hermitian $i \operatorname{Im} A$. As the singular values of $i \operatorname{Im} A$ are $\left\{\left|\lambda_{s}\right|\right\}$, we obtain

$$
\begin{aligned}
\operatorname{tr} \operatorname{Re} A & =\sum_{s} z_{s}^{\dagger}(\operatorname{Re} A) z_{s} \geq \sum_{s}\left|z_{s}^{\dagger}(i \operatorname{Im} A) z_{s}\right| \\
& =\sum_{s}\left|\lambda_{s}\right|=\|i \operatorname{Im} A\|_{1}=\|\operatorname{Im} A\|_{1} .
\end{aligned}
$$

\section{APPENDIX L: SOME RESULTS CONCERNING QUANTUM MULTIPARAMETER ESTIMATION}

This Appendix presents some interesting results concerning quantum multiparameter estimation, following Sec. VIII and assuming $1 \leq q \leq p<\infty$.

A crucial assumption in this paper is that $\mathcal{D}$, the set of influence operators, is not empty. While this assumption is not a problem for all the examples studied in this paper, the following theorem, generalizing a classical result by Stoica and Marzetta [70], can be used to verify the assumption.

Theorem 11.-D is not empty if and only if all the columns of $\partial \beta$ are in the range of the Helstrom information matrix $K$, viz.,

$$
K K^{+} \partial \beta=\partial \beta,
$$

where the superscript + denotes the Moore-Penrose pseudoinverse [71].

Proof.-We prove the "only if" part first. Assume that a $\delta \in \mathcal{D}$ exists. It satisfies $\langle S, \delta\rangle=\partial \beta$, and, therefore,

$$
u^{\top}\langle S, \delta\rangle v=\left\langle u^{\top} S, v^{\top} \delta\right\rangle=u^{\top}(\partial \beta) v,
$$

for any $u \in \mathbb{R}^{p}$ and $v \in \mathbb{R}^{q}$. The Cauchy-Schwarz inequality gives 


$$
\left|u^{\top}(\partial \beta) v\right|^{2} \leq\left(u^{\top} K u\right)\left(v^{\top}\langle\delta, \delta\rangle v\right)
$$

Now, suppose that $u$ is in the null space of $K$, such that $K u=0$, and pick $v=(\partial \beta)^{\top} u$. We obtain

$$
\left|u^{\top}(\partial \beta)(\partial \beta)^{\top} u\right|^{2} \leq 0
$$

which implies $(\partial \beta)^{\top} u=0$. As this condition must hold for any $u$ in the null space of $K$, each column of $\partial \beta$ must be orthogonal to the null space and, therefore, in the range of $K . K K^{+}$is the projection matrix into the range of $K$ [71], so Eq. (L1) holds.

The "if" part comes from the fact that, as long as Eq. (L1) holds,

$$
\delta=(\partial \beta)^{\top} K^{+} S
$$

satisfies $\langle\delta, I\rangle=0$ and $\langle S, \delta\rangle=K K^{+} \partial \beta=\partial \beta$ and is, therefore, an influence operator.

For an illustrative example, consider

$$
\theta=\left(\begin{array}{c}
\theta_{1} \\
\theta_{2}
\end{array}\right), \quad \partial \beta=\left(\begin{array}{l}
a \\
b
\end{array}\right)
$$

with the geometry depicted in Fig. 12. $S_{1}=0$ and $K_{11}=$ $\left\langle S_{1}, S_{1}\right\rangle=0$ at the singular point $\theta=\varphi$, meaning that

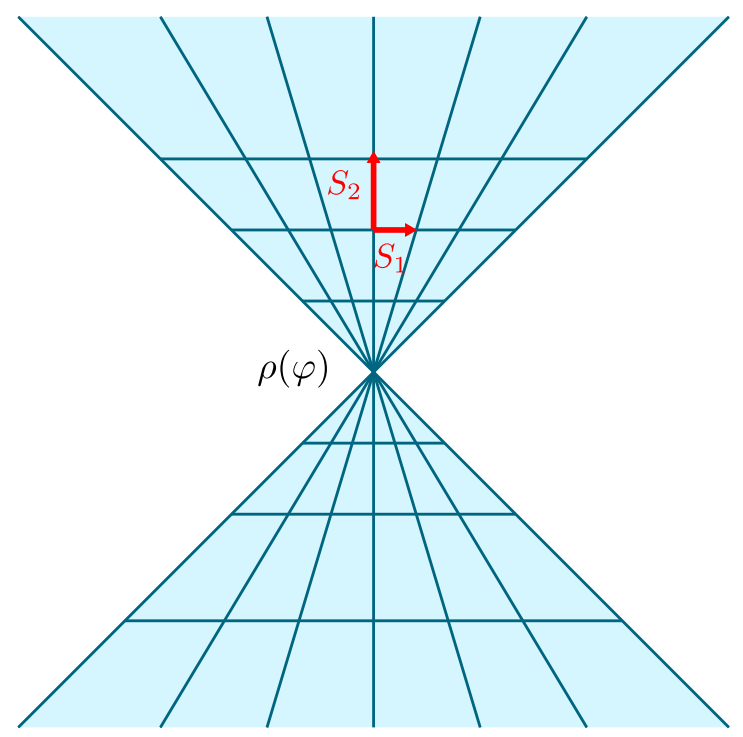

FIG. 12. The lines denote the Cartesian grid of $\theta_{1}$ and $\theta_{2}$. The distortion of the grid represents the geometry of $\rho\left(\theta_{1}, \theta_{2}\right)$. For example, the distance from one grid point $\left(\theta_{1}, \theta_{2}\right)$ to a neighboring $\left(\theta_{1}+\Delta, \theta_{2}\right)$ in the figure represents the distance between $\rho\left(\theta_{1}, \theta_{2}\right)$ and $\rho\left(\theta_{1}+\Delta, \theta_{2}\right) . S_{1}$ and $S_{2}$ are tangent vectors, and $K=\langle S, S\rangle$ is a metric that governs the infinitesimal distance between two neighboring $\rho$ 's. At the singular point $\rho(\varphi)$, $K_{11}=0$, the tangent space becomes a line in the $S_{2}$ direction, which forbids the existence of influence operators for certain $\partial \beta$.

$$
K(\varphi)=\left(\begin{array}{cc}
0 & 0 \\
0 & \left\langle S_{2}, S_{2}\right\rangle
\end{array}\right)
$$

The tangent space there becomes a line in the $S_{2}$ direction, and it is impossible for a $\delta$ to satisfy

$$
\langle S, \delta\rangle=\left(\begin{array}{c}
0 \\
\left\langle S_{2}, \delta\right\rangle
\end{array}\right)=\left(\begin{array}{l}
a \\
b
\end{array}\right),
$$

if $a \neq 0$.

If Eq. (L1) does not hold at certain values of $\theta$, Theorem 11 implies that an unbiased estimator of $\beta$ cannot exist there, and the GHB can be assumed to be infinite. Note, however, that a biased estimator may still be able to achieve a finite error.

Provided that Eq. (L1) holds, a pseudoinverse form of the Helstrom bound can be obtained.

Corollary 5.--If Eq. (L1) holds,

$$
\begin{gathered}
\delta_{\text {eff }}=(\partial \beta)^{\top} K^{+} S, \\
\left\langle\delta_{\text {eff }}, \delta_{\text {eff }}\right\rangle=(\partial \beta)^{\top} K^{+} \partial \beta, \\
\tilde{\mathrm{H}}=\operatorname{tr} W(\partial \beta)^{\top} K^{+} \partial \beta .
\end{gathered}
$$

Proof.-Equation (L5) is an influence operator and also a linear combination of $S$, so it is in the tangent space $\mathcal{T}$. By Theorem 1, it must be efficient. The other results follow from the fact $K^{+} K K^{+}=K^{+}$[71] and the definition of $\tilde{\mathrm{H}}$.

The original Helstrom bound is a simple consequence, generalizing the scalar version in Corollary 1.

Corollary 6.-If $K>0$,

$$
\begin{gathered}
\delta_{\text {eff }}=(\partial \beta)^{\top} K^{-1} S, \\
\left\langle\delta_{\text {eff }}, \delta_{\text {eff }}\right\rangle=(\partial \beta)^{\top} K^{-1} \partial \beta, \\
\tilde{\mathrm{H}}=\operatorname{tr} W(\partial \beta)^{\top} K^{-1} \partial \beta \equiv \mathrm{H} .
\end{gathered}
$$

Proof.-If $K>0, K^{-1}$ exists, $K^{+}=K^{-1}$, Eq. (L1) always holds, and the results follow from Corollary 5 .

Finally, we mention that the semidefinite program presented in Ref. [11] to evaluate the Holevo bound for $\beta=\theta$ and a nonsingular $K$ can be straightforwardly extended to the more general setup considered in this Appendix.

[1] C. W. Helstrom, Quantum Detection and Estimation Theory (Academic, New York, 1976).

[2] R. Demkowicz-Dobrzański, M. Jarzyna, and J. Kołodyński, Quantum Limits in Optical Interferometry, in Progress in 
Optics, Vol. 60, edited by E. Wolf (Elsevier, Amsterdam, 2015), Chap. 4, pp. 345-435.

[3] M. G. A. Paris, Quantum Estimation for Quantum Technology, Int. J. Quantum. Inform. 07, 125 (2009); V. Giovannetti, S. Lloyd, and L. Maccone, Advances in Quantum Metrology, Nat. Photonics 5, 222 (2011); M. Szczykulska, T. Baumgratz, and A. Datta, Multi-parameter Quantum Metrology, Adv. Phys.: X 1, 621 (2016); S. Pirandola, B. R. Bardhan, T. Gehring, C. Weedbrook, and S. Lloyd, Advances in Photonic Quantum Sensing, Nat. Photonics 12, 724 (2018); D. Braun, G. Adesso, F. Benatti, R. Floreanini, U. Marzolino, M. W. Mitchell, and S. Pirandola, Quantum-Enhanced Measurements without Entanglement, Rev. Mod. Phys. 90, 035006 (2018); L. Pezzé, A. Smerzi, M. K. Oberthaler, R. Schmied, and P. Treutlein, Quantum Metrology with Nonclassical States of Atomic Ensembles, Rev. Mod. Phys. 90, 035005 (2018); F. Albarelli, M. Barbieri, M. G. Genoni, and I. Gianani, A Perspective on Multiparameter Quantum Metrology: From Theoretical Tools to Applications in Quantum Imaging, Phys. Lett. A 384, 126311 (2020).

[4] M. Tsang, R. Nair, and X.-M. Lu, Quantum Theory of Superresolution for Two Incoherent Optical Point Sources, Phys. Rev. X 6, 031033 (2016).

[5] M. Tsang, Resolving Starlight: A Quantum Perspective, Contemp. Phys. 60, 279 (2019).

[6] M. I. Kolobov, The Spatial Behavior of Nonclassical Light, Rev. Mod. Phys. 71, 1539 (1999); Quantum Imaging, edited by M. I. Kolobov (Springer, New York, 2007); M. I. Kolobov and C. Fabre, Quantum Limits on Optical Resolution, Phys. Rev. Lett. 85, 3789 (2000); M. Ježek and Z. Hradil, Reconstruction of Spatial, Phase, and Coherence Properties of Light, J. Opt. Soc. Am. A 21, 1407 (2004); Z. Hradil, J. Řeháček, and L. L. Sánchez-Soto, Quantum Reconstruction of the Mutual Coherence Function, Phys. Rev. Lett. 105, 010401 (2010); M. A. Taylor and W. P. Bowen, Quantum Metrology and Its Application in Biology, Phys. Rep. 615, 1 (2016); M. Genovese, Real Applications of Quantum Imaging, J. Opt. 18, 073002 (2016); I. R. Berchera and I. P. Degiovanni, Quantum Imaging with Sub-Poissonian Light: Challenges and Perspectives in Optical Metrology, Metrologia 56, 024001 (2019); P.-A. Moreau, E. Toninelli, T. Gregory, and M. J. Padgett, Imaging with Quantum States of Light, Nat. Rev. Phys. 1, 367 (2019).

[7] A.S. Holevo, Probabilistic and Statistical Aspects of Quantum Theory (Edizioni della Normale, Pisa, Italy, 2011).

[8] M. Hayashi, Quantum Information Theory: Mathematical Foundation (Springer, Berlin, 2017).

[9] Asymptotic Theory of Quantum Statistical Inference: Selected Papers, edited by Masahito Hayashi (World Scientific, Singapore, 2005).

[10] H. Yuan and C.-H. Fred Fung, Quantum Parameter Estimation with General Dynamics, npj Quantum Inf. 3, 14 (2017); M. G. Genoni and T. Tufarelli, Non-orthogonal Bases for Quantum Metrology, J. Phys. A 52, 434002 (2019); K. Chabuda, J. Dziarmaga, T. J. Osborne, and R. Demkowicz-Dobrzański, Tensor-Network Approach for Quantum Metrology in Many-Body Quantum Systems, Nat. Commun. 11, 250 (2020); M. Fanizza, M. Rosati, M. Skotiniotis, J. Calsamiglia, and V. Giovannetti, Beyond the Swap Test: Optimal Estimation of Quantum State Overlap, Phys. Rev. Lett. 124, 060503 (2020).

[11] F. Albarelli, J. F. Friel, and A. Datta, Evaluating the Holevo Cramér-Rao Bound for Multiparameter Quantum Metrology, Phys. Rev. Lett. 123, 200503 (2019).

[12] M. Tsang, H. M. Wiseman, and C. M. Caves, Fundamental Quantum Limit to Waveform Estimation, Phys. Rev. Lett. 106, 090401 (2011); M. Guţă, Fisher Information and Asymptotic Normality in System Identification for Quantum Markov Chains, Phys. Rev. A 83, 062324 (2011).

[13] S. Ng, S.Z. Ang, T. A. Wheatley, H. Yonezawa, A. Furusawa, E. H. Huntington, and M. Tsang, Spectrum Analysis with Quantum Dynamical Systems, Phys. Rev. A 93, 042121 (2016).

[14] S. Zhou and L. Jiang, Modern Description of Rayleigh's Criterion, Phys. Rev. A 99, 013808 (2019).

[15] M. Tsang, Quantum Limit to Subdiffraction Incoherent Optical Imaging, Phys. Rev. A 99, 012305 (2019).

[16] O. Gühne and G. Tóth, Entanglement Detection, Phys. Rep. 474, 1-75 (2009).

[17] Quantum State Estimation, edited by M. Paris and J. Rehacek (Springer-Verlag, Berlin, 2004); A. I. Lvovsky and M. G. Raymer, Continuous-Variable Optical Quantum-State Tomography, Rev. Mod. Phys. 81, 299 (2009); R. Horodecki, P. Horodecki, M. Horodecki, and K. Horodecki, Quantum Entanglement, Rev. Mod. Phys. 81, 865 (2009); R. Filip, Overlap and Entanglement-Witness Measurements, Phys. Rev. A 65, 062320 (2002); T. A. Brun, Measuring Polynomial Functions of States, Quantum Inf. Comput. 4, 401 (2004), https://arxiv.org/abs/quant-ph/ 0401067; S. T. Flammia and Y.-K. Liu, Direct Fidelity Estimation from Few Pauli Measurements, Phys. Rev. Lett. 106, 230501 (2011); S. J. van Enk and C. W. J. Beenakker, Measuring $\operatorname{Tr} \rho^{n}$ on Single Copies of $\rho$ Using Random Measurements, Phys. Rev. Lett. 108, 110503 (2012).

[18] P. Horodecki, Measuring Quantum Entanglement without Prior State Reconstruction, Phys. Rev. Lett. 90, 167901 (2003).

[19] I. A. Ibragimov and R.Z. Has'minskii, Statistical Estimation: Asymptotic Theory (Springer, New York, 1981).

[20] P. J. Bickel, C. A. J. Klaassen, Y. Ritov, and J. A. Wellner, Efficient and Adaptive Estimation for Semiparametric Models (Springer, New York, 1993).

[21] A. A. Tsiatis, Semiparametric Theory and Missing Data (Springer, New York, 2006).

[22] W. K. Newey, Semiparametric Efficiency Bounds, J. Appl. Econom. 5, 99 (1990).

[23] Statistical Challenges in Modern Astronomy V, edited by E. D. Feigelson and J. Babu (Springer-Verlag, New York, 2012).

[24] M. Tsang, Semiparametric Estimation for Incoherent Optical Imaging, Phys. Rev. Research 1, 033006 (2019).

[25] A. S. Holevo, Commutation Superoperator of a State and Its Applications to the Noncommutative Statistics, Rep. Math. Phys. 12, 251 (1977).

[26] S.-i. Amari and H. Nagaoka, Methods of Information Geometry (American Mathematical Society/Oxford University Press, Providence, 2000).

[27] A. Uhlmann, Density Operators as an Arena for Differential Geometry, Rep. Math. Phys. 33, 253 (1993); S. L. 
Braunstein and C. M. Caves, Statistical Distance and the Geometry of Quantum States, Phys. Rev. Lett. 72, 3439 (1994); I. Bengtsson and K. Życzkowski, Geometry of Quantum States (Cambridge University Press, Cambridge, England, 2006); J.S. Sidhu and P. Kok, Geometric Perspective on Quantum Parameter Estimation, AVS Quantum Sci. 2, 014701 (2020).

[28] R. Demkowicz-Dobrzanski, W. Gorecki, and M. Guta, Multi-parameter Estimation beyond Quantum Fisher Information, arXiv:2001.11742.

[29] J. Kahn and M. Guţă, Local Asymptotic Normality for Finite Dimensional Quantum Systems, Commun. Math. Phys. 289, 597 (2009).

[30] R. D. Gill and M. Guţă, On Asymptotic Quantum Statistical Inference, in From Probability to Statistics and Back: HighDimensional Models and Processes-A Festschrift in Honor of Jon A. Wellner, Collections, Vol. 9, edited by M. Banerjee, F. Bunea, J. Huang, V. Koltchinskii, and M. H. Maathuis (Institute of Mathematical Statistics, Beachwood, OH, 2013), pp. 105-127; K. Yamagata, A. Fujiwara, and R. D. Gill, Quantum Local Asymptotic Normality Based on a New Quantum Likelihood Ratio, Ann. Stat. 41, 2197 (2013); Y. Yang, G. Chiribella, and M. Hayashi, Attaining the Ultimate Precision Limit in Quantum State Estimation, Commun. Math. Phys. 368, 223 (2019).

[31] M. D. Schwartz, Quantum Field Theory and the Standard Model (Cambridge University Press, Cambridge, England, 2014); P. M. Chaikin and T. C. Lubensky, Principles of Condensed Matter Physics (Cambridge University Press, Cambridge, England, 1995); H. Haken and H.C. Wolf, Molecular Physics and Elements of Quantum Chemistry: Introduction to Experiments and Theory, 2nd ed., Advanced Texts in Physics (Springer-Verlag, Berlin, 2004); S. Bravyi, D. Gosset, and R. Movassagh, Classical Algorithms for Quantum Mean Values, arXiv:1909.11485.

[32] Yu. Watanabe, T. Sagawa, and M. Ueda, Optimal Measurement on Noisy Quantum Systems, Phys. Rev. Lett. 104, 020401 (2010); Uncertainty Relation Revisited from Quantum Estimation Theory, Phys. Rev. A 84, 042121 (2011).

[33] I. Bloch, J. Dalibard, and W. Zwerger, Many-Body Physics with Ultracold Gases, Rev. Mod. Phys. 80, 885 (2008); I. M. Georgescu, S. Ashhab, and F. Nori, Quantum Simulation, Rev. Mod. Phys. 86, 153 (2014).

[34] C. Stein, Efficient Nonparametric Testing and Estimation, in Proceedings of the Third Berkeley Symposium on Mathematical Statistics and Probability, Vol. 1: Contributions to the Theory of Statistics (University of California Press, Berkeley, 1956), pp. 187-195.

[35] A. Carollo, B. Spagnolo, A. A. Dubkov, and D. Valenti, On Quantumness in Multi-parameter Quantum Estimation, J. Stat. Mech. (2019) 094010; Erratum: On Quantumness in Multi-parameter Quantum Estimation, J. Stat. Mech. (2020) 029902(E).

[36] J. Suzuki, Y. Yang, and M. Hayashi, Quantum State Estimation with Nuisance Parameters, arXiv:1911.02790.

[37] L. Debnath and P. Mikusiński, Introduction to Hilbert Spaces with Applications (Elsevier, Amsterdam, 2005).

[38] M. Reed and B. Simon, Methods of Modern Mathematical Physics. I: Functional Analysis (Academic, San Diego, 1980).
[39] S. Ragy, M. Jarzyna, and R. Demkowicz-Dobrzaski, Compatibility in Multiparameter Quantum Metrology, Phys. Rev. A 94, 052108 (2016).

[40] H. Nagaoka, A New Approach to Cramér-Rao Bounds for Quantum State Estimation, IEICE Technical Report No. IT 89-42, pp. 9-14 (1989), reprinted in Ref. [9], Chap. 8, pp. 100-112.

[41] More precisely, $\tilde{\beta}$ is the unique Riesz-Fréchet representation [38] of $D_{h} \beta$ as a continuous linear functional of $h \in \mathcal{Y}, \delta$ is that for $h \in \mathcal{Z} \subset \mathcal{Y}$, and $\delta_{\text {eff }}$ is that for $h \in \mathcal{T} \subseteq \mathcal{Z} \subset \mathcal{Y}$ [20,38]. The existence of each relies on $D_{h} \beta$ being continuous with respect to $h$ in each domain, so the existence of $\tilde{\beta}$ implies that of $\delta$ and $\delta_{\text {eff }}$.

[42] A. S. Holevo, Quantum Systems, Channels, Information (de Gruyter, Berlin, 2012).

[43] M. Tomamichel and M. Hayashi, A Hierarchy of Information Quantities for Finite Block Length Analysis of Quantum Tasks, IEEE Trans. Inf. Theory 59, 7693 (2013); K. Li, Second-Order Asymptotics for Quantum Hypothesis Testing, Ann. Stat. 42, 171 (2014); M. Tomamichel, Quantum Information Processing with Finite Resources: Mathematical Foundations (Springer, Cham, 2016).

[44] E. T. Jaynes, Information Theory and Statistical Mechanics. II, Phys. Rev. 108, 171 (1957).

[45] C. Gogolin and J. Eisert, Equilibration, Thermalisation, and the Emergence of Statistical Mechanics in Closed Quantum Systems, Rep. Prog. Phys. 79, 056001 (2016).

[46] T. Kinoshita, T. Wenger, and D.S. Weiss, A Quantum Newton's Cradle, Nature (London) 440, 900 (2006).

[47] T. Langen, S. Erne, R. Geiger, B. Rauer, T. Schweigler, M. Kuhnert, W. Rohringer, I. E. Mazets, T. Gasenzer, and J. Schmiedmayer, Experimental Observation of a Generalized Gibbs Ensemble, Science 348, 207 (2015).

[48] T. Langen, R. Geiger, and J. Schmiedmayer, Ultracold Atoms Out of Equilibrium, Annu. Rev. Condens. Matter Phys. 6, 201 (2015).

[49] J. Beirlant, E. J. Dudewicz, L. Györfi, and E. C. van der Meulen, Nonparametric Entropy Estimation: An Overview, Int. J. Math. Stat. Sci. 6, 17 (1997); L. Paninski, Estimation of Entropy and Mutual Information, Neural Comput. 15, 1191 (2003); T. M. Cover and J. A. Thomas, Elements of Information Theory (Wiley, New York, 2006).

[50] R. Jozsa, M. Horodecki, P. Horodecki, and R. Horodecki, Universal Quantum Information Compression, Phys. Rev. Lett. 81, 1714 (1998).

[51] H. M. Wiseman and G. J. Milburn, Quantum Measurement and Control (Cambridge University Press, Cambridge, England, 2010).

[52] L. Mandel and E. Wolf, Optical Coherence and Quantum Optics (Cambridge University Press, Cambridge, England, 1995).

[53] L. M. Artiles, R. D. Gill, and M. I. Guţă, An Invitation to Quantum Tomography, J. R. Stat. Soc. Ser. B 67, 109 (2005).

[54] A. B. Tsybakov, Introduction to Nonparametric Estimation (Springer, New York, 2009).

[55] M. Tsang, Subdiffraction Incoherent Optical Imaging via Spatial-Mode Demultiplexing, New J. Phys. 19, 023054 (2017).

[56] J.W. Goodman, Introduction to Fourier Optics (McGraw-Hill, New York, 2004). 
[57] L. T. Hall, J. H. Cole, C. D. Hill, and L. C. L. Hollenberg, Sensing of Fluctuating Nanoscale Magnetic Fields Using Nitrogen-Vacancy Centers in Diamond, Phys. Rev. Lett. 103, 220802 (2009); M. D. Vidrighin, G. Donati, M. G. Genoni, X.-M. Jin, W. S. Kolthammer, M. S. Kim, A. Datta, M. Barbieri, and I. A. Walmsley, Joint Estimation of Phase and Phase Diffusion for Quantum Metrology, Nat. Commun. 5, 3532 (2014); D. Branford, C. N. Gagatsos, J. Grover, A. J. Hickey, and A. Datta, Quantum Enhanced Estimation of Diffusion, Phys. Rev. A 100, 022129 (2019).

[58] References $[4,24,55]$ use the symbol $L$ for the number of detected photons, which is stochastic, and $N$ for the expected number of detected photons. For optics models with Poisson statistics, the conditioning on the detected photon number does not introduce any significant difference to the theory.

[59] In practice, a histogram of the photon counts at the detectors provides sufficient statistics for the estimators, and the photons do not need to be resolved individually [24].

[60] NIST Handbook of Mathematical Functions, edited by F. W. J. Olver, D. W. Lozier, R. F. Boisvert, and C. W. Clark (NIST and Cambridge University Press, Cambridge, England, 2010).

[61] M. Tsang, Subdiffraction Incoherent Optical Imaging via Spatial-Mode Demultiplexing: Semiclassical Treatment, Phys. Rev. A 97, 023830 (2018).

[62] K. A. G. Bonsma-Fisher, W.-K. Tham, H. Ferretti, and A. M. Steinberg, Realistic Sub-Rayleigh Imaging with PhaseSensitive Measurements, New J. Phys. 21, 093010 (2019).

[63] E. Bisketzi, D. Branford, and A. Datta, Quantum Limits of Localisation Microscopy, New J. Phys. 21, 123032 (2019); C. Lupo, Z. Huang, and P. Kok, Quantum Limits to Incoherent Imaging Are Achieved by Linear Interferometry, Phys. Rev. Lett. 124, 080503 (2020).

[64] S. Alipour and A. T. Rezakhani, Extended Convexity of Quantum Fisher Information in Quantum Metrology, Phys. Rev. A 91, 042104 (2015).

[65] V. P. Belavkin and B. A. Grishanin, Optimum Estimation in Quantum Channels by the Generalized Heisenberg Inequality Method, Prob. Peredachi Inf. 9, 44 (1973), http://mi .mathnet.ru/eng/ppi907.

[66] J. Suzuki, Explicit Formula for the Holevo Bound for TwoParameter Qubit-State Estimation Problem, J. Math. Phys. (N.Y.) 57, 042201 (2016); Information Geometrical Characterization of Quantum Statistical Models in Quantum Estimation Theory, Entropy 21, 703 (2019).

[67] Bayesian Bounds for Parameter Estimation and Nonlinear Filtering/Tracking, edited by Harry L. Van Trees and K. L.
Bell (Wiley-IEEE, Piscataway, 2007); M. P. Schützenberger, A Generalization of the Fréchet-Cramér Inequality to the Case of Bayes Estimation, Bull. Am. Math. Soc. 63, 142 (1957); H. L. Van Trees, Detection, Estimation, and Modulation Theory, Part I. (Wiley, New York, 2001); R. D. Gill and B. Y. Levit, Applications of the Van Trees Inequality: A Bayesian Cramér-Rao Bound, Bernoulli 1, 59 (1995); S. Personick, Application of Quantum Estimation Theory to Analog Communication over Quantum Channels, IEEE Trans. Inf. Theory 17, 240 (1971); M. Hayashi, Comparison between the Cramer-Rao and the Mini-max Approaches in Quantum Channel Estimation, Commun. Math. Phys. 304, 689 (2011); J. Liu and H. Yuan, Valid Lower Bound for All Estimators in Quantum Parameter Estimation, New J. Phys. 18, 093009 (2016); K. Chabuda, I. D. Leroux, and R. Demkowicz-Dobrzański, The Quantum Allan Variance, New J. Phys. 18, 083035 (2016); J. Rubio and J. Dunningham, Quantum Metrology in the Presence of Limited Data, New J. Phys. 21, 043037 (2019); Bayesian Multiparameter Quantum Metrology with Limited Data, Phys. Rev. A 101, 032114 (2020).

[68] Y. Tsuda and K. Matsumoto, Quantum Estimation for Nondifferentiable Models, J. Phys. A 38, 1593 (2005); V. Giovannetti, S. Lloyd, and L. Maccone, Quantum Measurement Bounds beyond the Uncertainty Relations, Phys. Rev. Lett. 108, 260405 (2012); M. Tsang, Ziv-Zakai Error Bounds for Quantum Parameter Estimation, Phys. Rev. Lett. 108, 230401 (2012); D. W. Berry, M. Tsang, M. J. W. Hall, and H. M. Wiseman, Quantum Bell-Ziv-Zakai Bounds and Heisenberg Limits for Waveform Estimation, Phys. Rev. X 5, 031018 (2015); X.-M. Lu and M. Tsang, Quantum Weiss-Weinstein Bounds for Quantum Metrology, Quantum Sci. Technol. 1, 015002 (2016); M. J. W. Hall and H. M. Wiseman, Does Nonlinear Metrology Offer Improved Resolution? Answers from Quantum Information Theory, Phys. Rev. X 2, 041006 (2012); R. Nair, Fundamental Quantum Limits in Optical Metrology from Rate-Distortion Theory, J. Phys. A 51, 434001 (2018).

[69] T. F. Jordan, Operators for Observables in Quantum Optics, Phys. Lett. 11, 289 (1964); C. L. Mehta and E. C. G. Sudarshan, Relation between Quantum and Semiclassical Description of Optical Coherence, Phys. Rev. 138, B274 (1965).

[70] P. Stoica and T. L. Marzetta, Parameter Estimation Problems with Singular Information Matrices, IEEE Trans. Signal Process. 49, 87 (2001).

[71] G. H. Golub and C. F. Van Loan, Matrix Computations (Johns Hopkins University Press, Baltimore, 2013). 